\title{
Unsteady Aerodynamics of Deformable Thin Airfoils
}

\author{
William Paul Walker \\ Thesis submitted to the Faculty of the \\ Virginia Polytechnic Institute and State University \\ in partial fulfillment of the requirements for the degree of \\ Master of Science \\ in \\ Aerospace Engineering
}

Mayuresh J. Patil, Chair

William J. Devenport

Robert A. Canfield

August 5, 2009

Blacksburg, Virginia

Keywords: Unsteady Aerodynamics, Deformable Airfoils

Copyright 2009, William Paul Walker 


\title{
Unsteady Aerodynamics of Deformable Thin Airfoils
}

\author{
William Paul Walker
}

(ABSTRACT)

Unsteady aerodynamic theories are essential in the analysis of bird and insect flight. The study of these types of locomotion is vital in the development of flapping wing aircraft. This paper uses potential flow aerodynamics to extend the unsteady aerodynamic theory of Theodorsen and Garrick (which is restricted to rigid airfoil motion) to deformable thin airfoils. Frequency-domain lift, pitching moment and thrust expressions are derived for an airfoil undergoing harmonic oscillations and deformation in the form of Chebychev polynomials. The results are validated against the time-domain unsteady aerodynamic theory of Peters. A case study is presented which analyzes several combinations of airfoil motion at different phases and identifies various possibilities for thrust generation using a deformable airfoil. 


\section{Contents}

1 Introduction $\quad 1$

2 Background and Objectives $r$

2.1 Literature Survey . . . . . . . . . . . . . . . . . . . 2

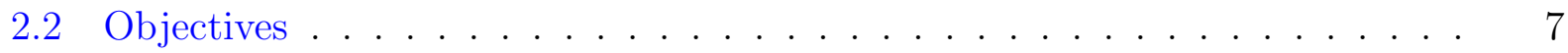

3 Aerodynamic Theory $\quad 9$

3.1 Lift and Pitching Moment Derivation . . . . . . . . . . . . . . . . 9

3.1.1 Mathematical Preliminaries . . . . . . . . . . . . . . 10

3.1.2 Noncirculatory Flow ..................... 13

3.1.3 Noncirculatory Pressure and Forces . . . . . . . . . . . . . . . . 16

3.1.4 Circulatory Flow . . . . . . . . . . . . . . . . . 19

3.1.5 Theodorsen's Function . . . . . . . . . . . . . . . . 24

3.1.6 Total Lift and Pitching Moment Expressions . . . . . . . . . . . . 26

3.2 Thrust Derivation . . . . . . . . . . . . . . . . . . . . . 29

3.2.1 Thrust due to Pressure . . . . . . . . . . . . . . . . . . 29

3.2.2 Thrust due to Leading Edge Suction . . . . . . . . . . . . . . . 30

3.2.3 Total Thrust Force . . . . . . . . . . . . . . . . . . . 32

4 Verification of Aerodynamic Theory $\quad 35$

4.1 Peters' Unsteady Airloads Theory . . . . . . . . . . . . . . . . . . 35

4.2 Application of Peters' Theory to Current Problem . . . . . . . . . . . . . 38 
5 Case Study $\quad 40$

5.1 Lift and Pitching Moment Results . . . . . . . . . . . . . . . . . . 40

5.1 .1 Reduction to Steady Thin Airfoil Theory . . . . . . . . . . . . . . . . 42

5.2 Thrust Results . . . . . . . . . . . . . . . . . . . 44

6 Conclusions and Future Work $\quad 55$

6.1 Conclusions . . . . . . . . . . . . . . . . . . . . 55

6.2 Future Work . . . . . . . . . . . . . . . . . 56

$\begin{array}{ll}\text { Bibliography } & 58\end{array}$

$\begin{array}{ll}\text { Appendix A } & 61\end{array}$ 


\section{List of Figures}

3.1 Chebychev Polynomials . . . . . . . . . . . . . . . . . . . . . . . . . 11

3.2 Joukowski mapping . . . . . . . . . . . . . . . . . . . . . . . . 14

3.3 Velocity due to generalized airfoil shape . . . . . . . . . . . . . 15

3.4 Vortex locations . . . . . . . . . . . . . . . . . . . . . . . . . 20

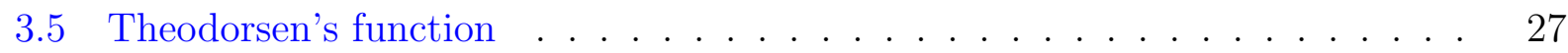

3.6 Fluid flow around leading edge . . . . . . . . . . . . . . . . . 31

5.1 Phase of lift relative to airfoil motion . . . . . . . . . . . . . . . 42

5.2 Phase of pitching moment relative to airfoil motion . . . . . . . . . . . 43

5.3 Thrust coefficient due to the first 5 airfoil shapes . . . . . . . . . . 46

5.4 Thrust coefficient due to $h$ and $\alpha \ldots \ldots \ldots \ldots \ldots . \ldots \ldots$

5.5 Thrust coefficient due to $h$ and $\kappa \ldots \ldots \ldots \ldots . \ldots \ldots 4$

5.6 Thrust coefficient due to $h$ and $\kappa_{2} \ldots \ldots \ldots \ldots \ldots \ldots$

5.7 Thrust coefficient due to $h$ and $\kappa_{3} \ldots \ldots \ldots \ldots \ldots \ldots$

5.8 Thrust coefficient due to $\alpha$ and $\kappa \ldots \ldots \ldots \ldots$

5.9 Thrust coefficient due to $\alpha$ and $\kappa_{2} \ldots \ldots \ldots \ldots \ldots \ldots \ldots \ldots \ldots \ldots \ldots \ldots$

5.10 Thrust coefficient due to $\alpha$ and $\kappa_{3} \ldots \ldots \ldots \ldots \ldots \ldots \ldots \ldots$

5.11 Thrust coefficient due to $\kappa$ and $\kappa_{2} \ldots \ldots \ldots \ldots \ldots \ldots \ldots \ldots \ldots$

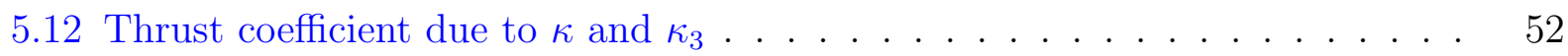

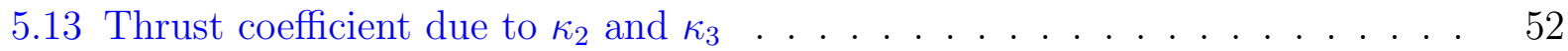

5.14 Thrust coefficient due to $h, \kappa_{2}$ and $\kappa_{3}\left(\phi_{\kappa 2}=-\pi / 2\right) \ldots \ldots \ldots 5$

5.15 Thrust coefficient due to $h, \kappa_{2}$ and $\kappa_{3}\left(\phi_{\kappa 2}=0\right) \ldots \ldots \ldots 53$ 
5.16 Thrust coefficient due to $h, \kappa_{2}$ and $\kappa_{3}\left(\phi_{\kappa 2}=\pi / 2\right) \ldots \ldots \ldots 4$

5.17 Thrust coefficient due to $h, \kappa_{2}$ and $\kappa_{3}\left(\phi_{\kappa 2}=\pi\right) \ldots \ldots \ldots$. . . . . 54

A.1 Functions $\mathrm{F}$ and $\mathrm{G}$ as a function of $1 / k \ldots \ldots . \ldots 63$ 


\section{List of Tables}

3.1 Velocity potential functions $\ldots \ldots \ldots \ldots$

3.2 Lift force components . . . . . . . . . . . . . . . . . . . . . . . . 28

3.3 Pitching moment components . . . . . . . . . . . . . . . . 28

3.4 Leading edge suction velocities . . . . . . . . . . . . . . . . . . . . . 31

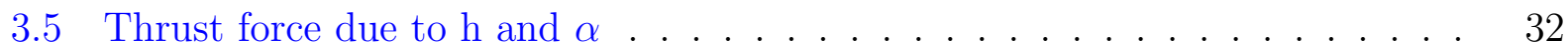

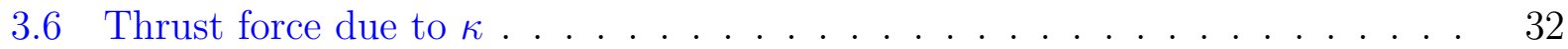

3.7 Thrust force due to $\kappa_{2} \ldots \ldots \ldots \ldots \ldots$

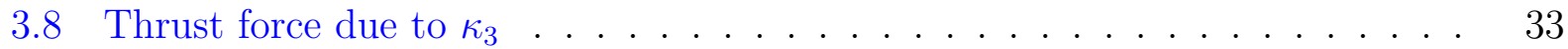

4.1 Lift force components . . . . . . . . . . . . . . . . . . . . . . . . . . 39

4.2 Pitching moment components . . . . . . . . . . . . . . . . . 39

5.1 Lift and pitching moment amplitude and phase terms . . . . . . . . . . 41 


\section{Nomenclature}

$\begin{array}{ll}A_{n} & \text { airfoil shape coefficient } \\ a & \text { joukowski mapping circle radius } \\ b & \text { semichord } \\ C(k) & \text { Theodorsen's function } \\ F(k) & \text { real component of Theodorsen's Function } \\ G(k) & \text { imaginary component of Theodorsen's function } \\ H_{n}(k) & \text { Hankel function } \\ h & \text { plunge variable } \\ \bar{h} & \text { plunge amplitude } \\ i & \text { imaginary number } \\ J_{n}(k) & \text { Bessel function of the first kind } \\ k & \text { reduced frequency } \\ L & \text { total lift } \\ L_{C} & \text { circulatory lift } \\ L_{N C} & \text { noncirculatory lift } \\ M_{N} & \text { total Pitching moment } \\ M_{C} & \text { circulatory Pitching moment } \\ M_{N C} & \text { noncirculatory Pitching moment } \\ p_{\infty} & \text { free stream pressure } \\ & \\ & \\ & \end{array}$


circulatory airfoil motion expression

leading edge suction

time

total thrust

Chebychev polynomials

thrust due to pressure

thrust due to leading edge suction

velocity in $\mathrm{x}$ direction

disturbance velocity in $\mathrm{x}$ direction

velocity in y direction

disturbance velocity in y direction

$\mathrm{x}$ location in unmapped plane

y location in unmapped plane

Bessel function of the second kind

$\mathrm{x}$ location in mapped plane

y location in mapped plane

point vortex circulation

pitch variable

pitch amplitude

wake vorticity per unit length

source/sink strength

curvature variable

curvature amplitude

higher Order shape terms

velocity potential

phase angle

density

frequency 


\section{Chapter 1}

\section{Introduction}

Micro Air Vehicles (MAVs) are a growing area of research. Flapping wing MAVs are similar to birds and insects, and are highly maneuverable. The aerodynamics of flapping wing aircraft is highly unsteady. Furthermore, during flight, bird and insect wings undergo significant aeroelastic deformations in addition to the prescribed rigid-body kinematics. Understanding the physics behind pitching, plunging and deforming airfoils will help in the design of flapping wing MAVs.

Analytical, frequency-domain, unsteady aerodynamics theory, such as Theodorsen [1] and Garrick [2] theory, has proven quite useful in understanding aeroelastic stability and thrust generation. However, Theodorsen and Garrick only modeled thin airfoils undergoing rigid body motion. Extending this unsteady aerodynamics theory to deformable airfoils will help in further understanding the unsteady aerodynamics and aeroelastic response of bird and insect flight, and help in improving the design of MAVs.

Inviscid potential flow theory may not accurately model flows at low Reynolds numbers or flows which have significant viscous effects, but it will help in increasing fundamental understanding of the unsteady aerodynamic flow and aeroelasticity of flapping wing MAVs. Furthermore, potential flow theory can serve as a check for verifying other more complex/computational codes and methods used in design. 


\section{Chapter 2}

\section{Background and Objectives}

\subsection{Literature Survey}

Much research has been done in unsteady wing and airfoil theory. Theodorsen used two dimensional elementary flows, solutions to Laplace's equation, to develop the velocity potential functions for a pitching and plunging flat plate with a flap[1]. The flow around a flat plate was modeled using Joukowski transformation which mapped the flow around a circle to flow around a flat plate. The source/sink and vortex flows were used to satisfy boundary conditions and Bernoulli's equation was used to obtain the unsteady airloads on a thin oscillating airfoil with a flap. Theodorsen assumed small perturbations, which implies a flat wake behind the airfoil extending to infinity. The airfoil motion was restricted to be harmonic. This assumption allowed the vortex sheet extending from the trailing edge to infinity to be integrated leading to a solution in the form of Bessel functions. Through this solution, Theodorsen showed that the lift due to circulation was a function of the reduced frequency. Theodorsen's function is useful in describing the effect of the wake on the airloads as a function of reduced frequency.

Garrick extended Theodorsen theory to develop the thrust force generated by a flat

plate in unsteady flow[2]. Garrick approached the problem in two different ways. First, the problem was solved using the principle of conservation of energy. The energy of the structural 
motion, which is the energy required to maintain the oscillations must be equal to the energy of the wake plus the energy of the propulsive force. This energy method involved calculating the average work done by the structural motion and the average energy in the wake over one period of oscillation. These average energies are used to solve for the average energy done by the propulsive force which is given as the average propulsive force multiplied by the velocity. However, this method yields only the average propulsive force over a period of oscillation. In actuality, the propulsive force is changing with time in a harmonic manner just like the lift and pitching moment. Garrick also calculated the propulsive force directly by calculating the leading edge suction and the component of the pressure in the horizontal direction.

The leading edge suction on an airfoil results from the flow reaching the stagnation point, and turning to go around the leading edge. von Kármán and Burgers showed the leading edge suction velocity on a flat plate by developing a function for the vorticity at the leading edge and finding the limit as leading edge radius approaches zero[3]. The leading edge suction force can be derived from the Blasius formula, as shown in Katz and Plotkin[4].

The second thrust component is the force from the pressure on the airfoil in the horizontal direction. Garrick showed that harmonic plunging will always lead to a positive propulsive force. This is a useful tool in the development of MAV's and the study of bird and insect flight.

The problem of an airfoil that is initially at rest and started abruptly was solved by Wagner[5]. For an airfoil at constant angle of attack, the lift starts at 50\% of the steady lift and asymtotically approaches the steady lift. This is due to the shed vortex initially after the airfoil starts moving. As the airfoil moves away from the initial location, the bound circulation gradually approaches a value.

The problem of an airfoil entering a sharp edged gust was investigated by Küssner[7]. Küssner developed a function to show how the lift changes as a function of time as an airfoil is entering a gust, which was similar to Wagner's function. The airfoil entering a gust is slightly different than an abrupt start of motion. This is because the effective change in angle of attack as the airfoil suddenly enters the gust acts over only part of the airfoil until 
the entire airfoil is inside the region being effected by the gust, while the abrupt start of an airfoil acts over the entire airfoil.

The relationship between Wagner's and Theodorsen's function was investigated by Garrick[6]. He also showed relationships between Theodorsen's function and Küssner's function[7].

The unsteady aerodynamics of an airfoil in non-uniform motion was addressed by von Kármán and Sears[8]. This theory derived the formulae for lift and pitching moment for general non-uniform motion, unlike theories by Theodorsen, Wagner, and Küssner which addressed specific flow situations. The theory shows the lift and pitching moment each to be a sum of three components, quasi-steady lift, apparent mass, and wake vorticity contribution. The equations for lift and pitching moment were applied to specific flow situations and shown to match theories by Theodorsen, Wagner, and Küssner. Sears later applied Heaviside's operators to obtain more convenient results of this general theory[9].

The work of Theodorsen and Garrick involves the unsteady aerodynamics of oscillating wings with a constant free-stream velocity. The problem of unsteady aerodynamics on thin airfoils with a non constant free stream velocity has been investigated by a number of researchers. Isaacs derived a solution for the lift on an airfoil in a non constant free-stream[10], and investigated in particular the case of a rotary wing aircraft in forward flight[11]. The rotary wing case applied only to an airfoil at constant angle of attack. This was done by assuming the free-stream velocity to be a constant value plus a sinusoidal term. However, Isaacs did not account for the wake moving downward due to the inflow of the rotor. This wake shape would be a type of distorted helix shape.

The problem of the rotary wing was also investigated by Loewy[12]. Loewy solved the problem of a rotary wing with no forward velocity, as in a helicopter in hover. Therefore, for each spanwise location, the free stream velocity is constant in time. Loewy developed a solution for this problem similar to Theodorsen theory. Along with the usual assumption of small disturbances, Loewy assumed the chord, amplitude of oscillation in effective angle of attack and relative airspeed vary slowly enough with span to say that the aerodynamics 
at each blade radius are essentially the same on either side. Essentially this assumption eliminated three dimensional effects. The solution by Loewy allows for all the unsteady motion of Theodorsen theory. The difference between Theodorsen theory and Loewy's theory is the wake shape. The wake for a rotary wing in hover is a helix shape. Loewy modeled this in two dimensions with a layered wake, where the distance between the layers depends on the inflow velocity to the rotor, the number of blades in the rotor, and the period of oscillation of the rotor. This wake model resulted in a function of frequency similar to Theodorsen theory. Loewy showed that for the case of a rotary wing in hover, the aerodynamic forces have the same form as that for non-rotating wings. Replacing Theodorsen's function with Loewy's function changes the problem from that of a thin airfoil in harmonic motion to that of a rotary wing, undergoing harmonic motion.

The non constant free-stream velocity problem was also addressed by Greenberg[13]. In his work, Greenberg developed an extension to Theodorsen theory to take into account the non constant free-stream, unlike Isaacs however, Greenberg made an assumption that wake was sinusoidal, such as the wake model used by Theodorsen. Therefore, the wake propagates downstream with a constant velocity. This allowed Greenberg to obtain a solution to the non constant free-stream problem for unsteady airfoil motions as well as just an airfoil at constant angle of attack. Greenberg showed, via a numerical example, that the use of the sinusoidal wake compared well to the model used by Isaacs.

There have been a number of investigations into relating the methods of Theodorsen, Garrick, Loewy, Greenberg, and Isaacs to the aerodynamics of bird and insect wings. Zbikowski[14] obtained a solution for unsteady flow in a form similar to von Kármán and Sears on a wing which is pitching, plunging, and can take into account non constant free-stream, similar to Greenberg's work. This solution was given in several different forms, including time and frequency domains. However, the wake is integrated to a certain point, which is a function of time, and not to infinity. This allows for a more general form of the solution to the problem investigated by Wagner[5].

Other work in the application of unsteady flow of thin airfoils to bird and insect 
flight was done by Azuma and Okamoto[15]. Insects such as dragonflies have corrugated shaped wings, which pitch and plunge, while maintaining the airfoil shape. Their work was an extension of Theodorsen theory to a combination of flat plate airfoils to give a corrugated wing shape. The theory uses a set of flat plates attached end to end at various angles. This allows for any number of airfoil shapes, and as the number of elements used increases, a smooth airfoil, such as a curved plate, can be obtained.

Further study into the energy of the harmonic oscillating airfoil system, and it's relationship to aeroelastic systems, has been presented by Patil[16]. Using the methods derived by Theodorsen and Garrick, Patil expanded the view of energy transfer in aeroelastic analyses. The flow of energy was previously viewed as flowing between the structural motion and the flow. However, the energy transfer to the flow has two destinations, the wake and energy into propulsive force. This was also shown by Garrick in his energy approach to deriving the propulsive force for a harmonic oscillating wing. Using the expressions for energy, and conservation of energy, Patil showed that there are three possible energy transfer modes, which depend on the direction of the energy flow. This led to the conclusion that, for a flutter mode, the horizontal force must be a drag, and for a thrust producing mode, the oscillations are damped, meaning the energy must come from the structure to maintain oscillations. Also it is possible to have a damped mode, which also produces drag, in which case all the energy transfers to the wake. This proves very helpful in the study of aeroelasticity for thin airfoils in an unsteady flow.

Aerodynamics of deformable airfoils was investigated by Peters[17]. The theory developed by Peters is a general theory for the airloads on a deformable wing of arbitrary shape with any form of free stream velocity. Peters' theory is comprised of two parts, the airloads theory and the wake model[18]. The airloads theory was developed for a arbitrary airfoil shape in terms of the Chebychev polynomials. Combinations of these polynomial shapes can lead to any possible shape desired because they are a complete set of functions. The wake model can be any model desired depending on the type of flow considered. Peters shows by using wake models from Theodorsen, Garrick, Wagner and others that these classical theories 
for a rigid airfoil motion can be completely recovered with his theory.

The aerodynamics of deformable airfoils was also investigated by Johnston[19]. Johnston applied the general unsteady airfoil theory of von Kármán and Sears to a general deforming camberline. Examples of different deformation shapes such as NACA 4-series camberlines and curved leading and trailing edges (not hinged) were given.

There has been much more work in the field of unsteady flow on airfoils, including work by Glegg and Devenport, which investigates the problem of unsteady loading on an airfoil with thickness[20]. Their work uses conformal mapping to get any desired airfoil shape, instead of just a flat plate such as Theodorsen. The forces were derived from the Blasius theorem. Robert Jones investigated the unsteady aerodynamics of a wing with finite $\operatorname{span}[21]$. Jones' work comprised of correcting the angle of attack for the downwash velocity generated by three dimensional effects. Other work has been done investigating the problem of unsteady compressible flows, including work by Hernandes and Soviero[22].

\subsection{Objectives}

The aim of the present research is to extend the unsteady aerodynamic theories of Theodorsen[1] and Garrick[2] to deformable airfoils. Analytic expressions for lift, thrust and pitching moment of a thin airfoil undergoing harmonic motion (including harmonically changing deformation) is developed in the frequency domain. To be able to represent a general deformable airfoil, the airfoil deformation can be represented in terms of an infinite series of

polynomials. For the present research, Chebychev polynomials are used in order to verify with Peters' theory[17]; the first two polynomials account for rigid body motion and the rest account for the deformations.

The extended theory is verified by confirming that it converges to the solution for the rigid body motion given by Theodorsen[1] and Garrick[2]and then compared to a time domain solution developed by Peters[17] for deformable airfoils.

Results are presented for the aerodynamic load on an airfoil undergoing harmonic 
motion/deformation corresponding to the first few deformation shapes. The lift and pitching moment are calculated for harmonic oscillations corresponding to each individual polynomial shape. The lift and pitching moment are linear and thus one can calculate the results for oscillations made up of any combination of deformation shapes (with any relative magnitude and phase) by superposition. The thrust is a nonlinear result and results are presented for oscillation in just one shape as well as for various combinations of two deformation shapes (with any relative magnitude and phase). 


\section{Chapter 3}

\section{Aerodynamic Theory}

The deformable unsteady airloads theory presented in this research is based on earlier theories developed by Theodorsen[1] and Garrick[2]. The theory is verified by the unsteady airloads theory developed by Peters[18]. The derivation of the aerodynamic loads on a thin oscillating deformable airfoil is presented in this chapter.

\subsection{Lift and Pitching Moment Derivation}

Theodorsen's method for thin oscillating airfoils is based on ideal flow in two dimensions. The elementary flows, which are solutions to Laplace's equation, are combined to develop the flow due to the disturbance of the airfoil in the flow, the flow due to circulation on the airfoil, and the flow due to vortices in the wake. The sum of elementary flow solutions is itself a solution to Laplace's equation, because it is a linear differential equation. This allows the superposition of flows, which allows for the separate development of the airloads due to circulation and the airloads due to accelerations. Laplace's equation is given as,

$$
\nabla^{2} \phi=0
$$

where, $\phi$ is the velocity potential. 
The airloads model is developed for a thin airfoil, essentially a flat plate. The method in this derivation uses conformal mapping to develop the flow around the flat plate from the flow around a circle in two dimensions. First, the noncirculatory flow is developed with a source-sink pair sheet distributed along the chord. The strength of the sheet is derived from the boundary condition of no flow through the surface of the airfoil. The circulatory flow is derived by using a vortex sheet on the airfoil and in the wake, which extends to infinity. The strength of this sheet is determined by the Kutta condition.

\subsubsection{Mathematical Preliminaries}

The Joukowski conformal mapping function, given below, is used to map the circle of radius, $b$, centered at the origin to a flat plate of semichord, $b$. The mapping function maps all the points outside of the circle to a location outside the the flat plate, points inside the circle to a location outside the flat plate, but on a different Riemann surface, and the function maps points on the circle directly to the flat plate[23].

$$
x+i y=X+i Y+\frac{b^{2}}{X+i Y}
$$

The airfoil motion/deformation is represented by plunge, pitch, and different forms of camber/deformation shapes. These shapes are defined by a set of complete orthogonal polynomials so that one can represent any deformed shape by considering enough polynomials in the solution. The orthogonal polynomials chosen for the present work are the Chebychev polynomials. This was done to verify the results of this derivation with those of Peters. The Chebychev polynomials are given as, 


$$
\begin{aligned}
T_{0}(x) & =1 \\
T_{1}(x) & =x \\
T_{n+1}(x) & =2 x T_{n}(x)-T_{n-1}(x)
\end{aligned}
$$

where, $x$ is the non-dimensional variable along the chord varying from -1 at the leading edge to 1 at the trailing edge. The first five polynomials, given below, and shown in Figure 3.1, are used in this derivation. However, this is a general derivation, and any number of polynomials can be used to understand the unsteady aerodynamics of more complicated deformation shapes.

$$
\begin{aligned}
& T_{0}(x)=1 \\
& T_{1}(x)=x \\
& T_{2}(x)=2 x^{2}-1 \\
& T_{3}(x)=4 x^{3}-3 x \\
& T_{4}(x)=8 x^{4}-8 x^{2}+1
\end{aligned}
$$

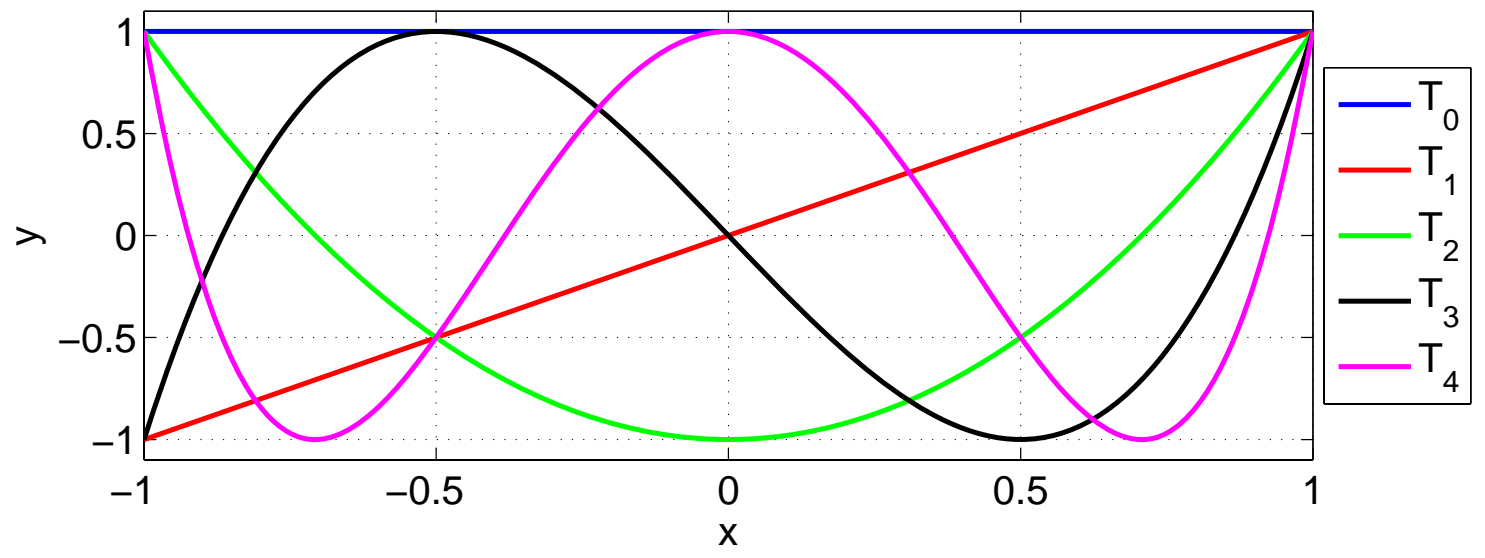

Figure 3.1: Chebychev Polynomials 
These polynomials are orthogonal on $-1 \leq x \leq 1$ with respect to a weight of $1 / \sqrt{1-x^{2}}$. There are other sets of functions which are directly orthogonal, such as the Legendre polynomials. Chebychev are used because the results will be compared to Peters' theory, which requires the use of Chebychev polynomials. The theory presented in this thesis does not have this requirement and thus any complete set of orthogonal functions can be used. The order of these polynomials determines the type of motion described by them. The first Chebychev polynomial is a constant in space, which represents vertical displacement. The time rate of change of vertical displacement is the plunge velocity. The second polynomial is a linear function, which represents a flat plate at an angle to the flow, which is the angle of attack. Likewise, the third polynomial is quadratic, which represents a quadratic camber. This results in a generalized airfoil shape in terms of the Chebychev polynomials is given as,

$$
y(x)=A_{0} T_{0}(x)+A_{1} T_{1}(x)+A_{2} T_{2}(x)+\ldots+A_{n} T_{n}(x)
$$

where, the $A_{n}$ 's are the magnitude of each polynomial contributing to the complete airfoil shape. This representation for a general airfoil shape is not very meaningful. The $A_{n}$ 's can be related to the classical airfoil shape variables, such as $h$ for plunge and $\alpha$ for pitch. $A_{0}$ is the plunge location, $h / b$. The second Chebychev polynomial is linear and corresponds to the slope of the airfoil, and therefore $A_{1}=\alpha$. The third Chebychev polynomial represents curvature (or quadratic camber), but the second derivative of the polynomial is not one. To give physical meaning to the shape, $A_{n}$ 's are written in terms of the physically relevant deformation variables by adding a scaling factor corresponding to the highest nonzero derivative. Therefore it can be seen that the scaling factor multiplied by the Chebychev polynomials is determined by the highest nonzero derivative. Therefore the airfoil deformation function is given as, 


$$
\begin{aligned}
y(x, t) & =\sum_{n=0}^{N} A_{n} T_{n} \\
A_{n} & =\frac{b^{n-1}}{\frac{\partial^{n} T_{n}}{\partial x^{n}}} \frac{\partial^{n} y_{n}}{\partial x^{n}} \\
y(x, t) & =\frac{h}{b} T_{0}+\alpha T_{1}+\frac{b \kappa}{4} T_{2}+\frac{b^{2} \kappa_{2}}{24} T_{3}+\frac{b^{3} \kappa_{3}}{192} T_{4}+\ldots+A_{N} T_{N}
\end{aligned}
$$

\subsubsection{Noncirculatory Flow}

The noncirculatory part of the flow is solved using sources and sinks. The velocity potential for the flow due to a source or a sink is given in as,

$$
\phi_{\text {source } / \operatorname{sink}}=\frac{\epsilon}{4 \pi} \log \left(X-X_{1}\right)^{2}+\left(Y-Y_{1}\right)^{2}
$$

where, $\epsilon$ is the strength of the source/sink, $X$ and $Y$ are non-dimensional spatial variables in the unmapped domain, and $X_{1}$ and $Y_{1}$ are the location of the point source/sink.

Placing a double source of strength $2 \epsilon$ at a point $\left(X_{1}, Y_{1}\right)$ and a double sink of strength $-2 \epsilon$ at $\left(X_{1},-Y_{1}\right)$, the source/sink pair velocity potential is found by summing the velocity potential expressions. The conformal mapping representation of the wing in the unmapped plane as a circle is shown in Figure 3.2. The velocity potential of the source/sink pair in the unmapped plane is given as,

$$
\phi_{\text {source } / \text { sink }}=\frac{\epsilon}{2 \pi} \log \frac{\left(X-X_{1}\right)^{2}+\left(Y-Y_{1}\right)^{2}}{\left(X-X_{1}\right)^{2}+\left(Y+Y_{1}\right)^{2}}
$$

$Y=\sqrt{1-X^{2}}$ and $Y_{1}=\sqrt{1-X_{1}^{2}}$ for the upper surface of a circle. Thus, the velocity potential can be written as, 


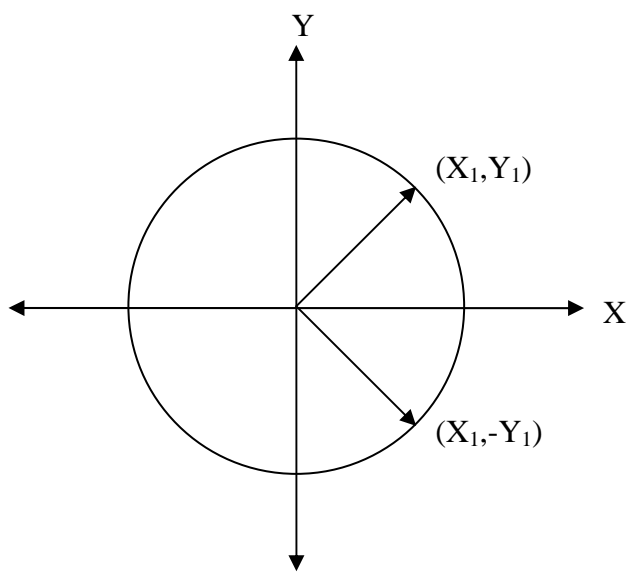

Figure 3.2: Joukowski mapping

$$
\phi=\frac{\epsilon}{2 \pi} \log \frac{\left(X-X_{1}\right)^{2}+\left(\sqrt{1-X^{2}}-\sqrt{1-X_{1}^{2}}\right)^{2}}{\left(X-X_{1}\right)^{2}+\left(\sqrt{1-X^{2}}+\sqrt{1-X_{1}^{2}}\right)^{2}}
$$

Constraining the equation to points only on the upper surface of the circle is helpful because these locations map directly to location on the airfoil in the Joukowski map. Constraining the Joukowski mapping function, Eq. (3.2), to the surface of the circle simplifies the relationship between unmapped and mapped locations.

$$
\begin{aligned}
x+i y & =X+i \sqrt{1-X^{2}}+\frac{1}{x+i \sqrt{1-X^{2}}} \\
& =X+i \sqrt{1-X^{2}}+\frac{1}{x+i \sqrt{1-X^{2}}} \frac{\left(x-i \sqrt{1-X^{2}}\right)}{\left(x-i \sqrt{1-X^{2}}\right)} \\
x & =2 X
\end{aligned}
$$

Therefore the expression in Eq. (3.9) gives the velocity potential expression on the surface of the airfoil, and can be written as, 


$$
\phi=\frac{\epsilon}{2 \pi} \log \frac{\left(x-x_{1}\right)^{2}+\left(\sqrt{1-x^{2}}-\sqrt{1-x_{1}^{2}}\right)^{2}}{\left(x-x_{1}\right)^{2}+\left(\sqrt{1-x^{2}}+\sqrt{1-x_{1}^{2}}\right)^{2}}
$$

Integrating the expression for the source/sink pair along the chord gives a general relationship for the velocity potential due to the source/sink pair sheet. This will be used to describe all noncirculatory flows depending on the value of the strength, $\epsilon(x, t)$.

$$
\phi=\frac{b}{2 \pi} \int_{-1}^{1} \epsilon(x, t) \log \frac{\left(x-x_{1}\right)^{2}+\left(\sqrt{1-x^{2}}-\sqrt{1-x_{1}^{2}}\right)^{2}}{\left(x-x_{1}\right)^{2}+\left(\sqrt{1-x^{2}}+\sqrt{1-x_{1}^{2}}\right)^{2}} d x_{1}
$$

The velocity generated by the source/sink pair must satisfy the no penetration boundary condition. Thus, the velocity must be equal and opposite to the component of free stream velocity minus the transverse velocity of motion of the airfoil at that point. Starting with a general shape of the airfoil the disturbance flow needed to counteract the free stream flow resembles Figure 3.3.

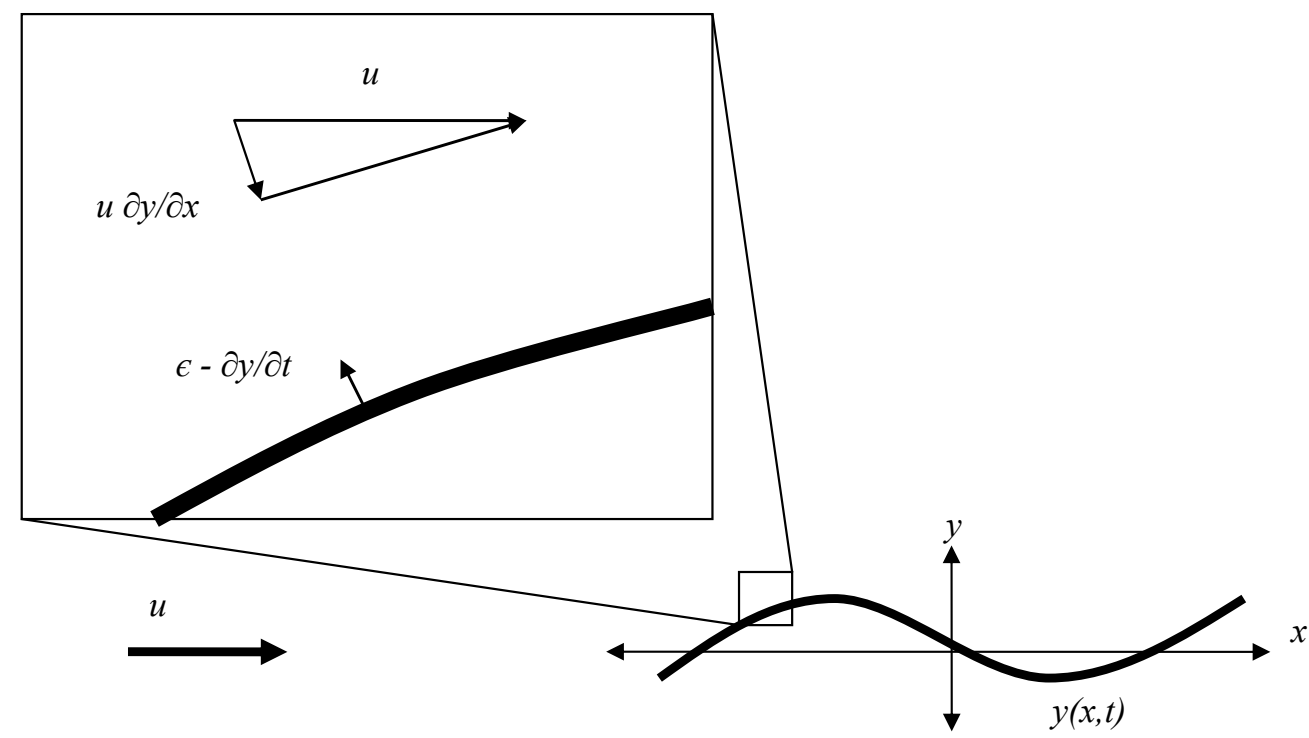

Figure 3.3: Velocity due to generalized airfoil shape 
The strength of the source/sink sheet is determined by the boundary condition that no flow penetrate the airfoil. Approaching the surface of the source/sink sheet, the velocity must be perpendicular to the surface, and at this point the velocity of the fluid flowing out of the source is the strength of the source[4]. Therefore, assuming small angles, the strength of the source/sink sheet for a generalized airfoil shape, is given by,

$$
\epsilon(x, t)=u \frac{\partial y}{\partial x}+b \frac{\partial y}{\partial t}
$$

where, $u$ is the free stream velocity.

The airfoil deformation expression, Eq. (3.6), can be substituted into the source/sink strength equation given above, and this strength can be substituted into the velocity potential equation, Eq. (3.12), leading to the potential for the noncirculatory flow as a function of $x$. The velocity potential expressions for the first five airfoil shapes are shown in Table 3.1, where the first two rows match Theodorsen[1]. These equations are functions of $x$ only and are valid for the upper surface of the airfoil. This is because the general equation for the velocity potential, Eq. (3.12), was constrained to the upper surface of the circle. The pressure on the airfoil due to these flows and the forces produced are derived in the following sections.

Table 3.1: Velocity potential functions

\begin{tabular}{cc}
\hline \hline & \\
$\phi_{h}=0$ & $\phi_{\dot{h}}=\dot{h} b \sqrt{1-x^{2}}$ \\
$\phi_{\alpha}=u \alpha b \sqrt{1-x^{2}}$ & $\phi_{\dot{\alpha}}=\frac{\dot{\alpha} b^{2}}{2} x \sqrt{1-x^{2}}$ \\
$\phi_{\kappa}=\frac{u b^{2} \kappa}{2} x \sqrt{1-x^{2}}$ & $\phi_{\kappa}=-\frac{b^{3} \dot{k}}{6}\left(1-x^{2}\right)^{3 / 2}$ \\
$\phi_{\kappa 2}=-\frac{u b^{3} \kappa_{2}}{24}\left(1-4 x^{2}\right) \sqrt{1-x^{2}}$ & $\phi_{\dot{\kappa} 2}=-\frac{b^{4} \kappa_{2}}{24} x\left(1-x^{2}\right)^{3 / 2}$ \\
$\phi_{\kappa 3}=-\frac{u b^{4} \kappa_{3}}{48} x\left(1-2 x^{2}\right) \sqrt{1-x^{2}}$ & $\phi_{\dot{\kappa} 3}=\frac{b^{5} \dot{\kappa}_{3}}{720}\left(1+6 x^{2}\right)\left(1-x^{2}\right)^{3 / 2}$ \\
\hline
\end{tabular}

\subsubsection{Noncirculatory Pressure and Forces}

The desired result is ultimately the lift force and pitching moment, which requires the pressure. Bernoulli's equation gives the pressure using the velocity potential equations previously 
developed. Bernoulli's equation can be written as,

$$
p_{0}=p+\frac{1}{2} \rho V^{2}+\rho \frac{\partial \phi}{\partial t}
$$

Solving this equation in the free-stream flow, the total pressure is given as,

$$
p_{0}=p_{\infty}+\frac{1}{2} \rho u^{2}
$$

where, $u$ is the free stream velocity. The Bernoulli equation is linearized for small disturbances by assuming that the disturbance velocities in the $x$ and $y$ directions, $u^{\prime}$ and $v^{\prime}$, are small.

$$
V=\left(u+u^{\prime}\right) i+v^{\prime} j \rightarrow V^{2}=\left(u^{2}+2 u u^{\prime}\right)
$$

The linearized Bernoulli equation is given as,

$$
p-p_{\infty}=-\rho\left(\frac{u}{b} \frac{\partial \phi}{\partial x}+\frac{\partial \phi}{\partial t}\right)
$$

The difference in the velocity potential between two locations in space is the integral of the velocity along the surface between these two locations,

$$
\phi_{2}-\phi_{1}=\int_{x_{1}}^{x_{2}} u^{\prime} d x
$$

where, $x_{1}$ and $x_{2}$ are locations in space and $\phi_{1}$ and $\phi_{2}$ are the velocity potential at those locations respectively. Considering only the disturbance potential, the disturbance flow at 
equal $X$ locations on the circle, but opposite $Y$ locations, must be the same. The integral of the velocity from some $X$ location on the upper side of the circle to the leading edge, and the integral from the leading edge to the same $X$ location on the lower half of the circle must be the same. Therefore the antisymmetric condition results in[23],

$$
\phi_{L E}-\phi_{U}(x)=\phi_{L}(x)-\phi_{L E}
$$

where, $\phi_{U}$ and $\phi_{L}$ are the velocity potentials on the upper and lower surfaces respectively. It is seen from Table 3.1 that the disturbance potential at the leading edge, $\phi_{L E}$, is zero, and therefore the pressure difference between the upper and lower surface is given as,

$$
p_{L}-p_{U}=-2 \rho\left(\frac{u}{b} \frac{\partial \phi_{U}}{\partial x}+\frac{\partial \phi_{U}}{\partial t}\right)
$$

The same equation results if the substitution of $Y=-\sqrt{1-X^{2}}$ was used in Eq. (3.8) to constrain points to the lower surface of the circle. The pressure difference would be the difference between the pressure Eq. (3.17) and the corresponding equation for pressure on the lower surface. Integrating the expression for the pressure difference across the airfoil gives the force acting on the airfoil. Likewise, integrating the pressure difference multiplied by the moment arm to the pivot point of the airfoil gives the pitching moment of the airfoil. The expressions for the first five shapes are shown below, where the pitching moment is calculated about the midchord. The pitch and plunge terms match with Theodorsen[1].

$$
\begin{aligned}
L_{N C} & =b \int_{-1}^{1}\left(p_{L}-p_{U}\right) d x=-\pi \rho b^{2}\left[u \dot{\alpha}+\ddot{h}-\frac{b^{2}}{8} \ddot{\kappa}\right] \\
M_{\alpha N C} & =b^{2} \int_{-1}^{1}\left(p_{L}-p_{U}\right) x d x=-\pi \rho b^{2}\left[-u^{2} \alpha+\frac{b^{2}}{8} \ddot{\alpha}-u \dot{h}+\frac{b^{3}}{4} \dot{\kappa}-\frac{b^{4}}{192} \ddot{\kappa}_{2}\right]
\end{aligned}
$$


The above equations give the airloads due to accelerations only. These equations are derived assuming small disturbances relative to the free stream and the boundary condition that no flow penetrate the surface of the airfoil.

\subsubsection{Circulatory Flow}

Thus far the model for the noncirculatory flow about a flat plate airfoil has been developed for unsteady motions in the shape of Chebychev polynomials. The model satisfies the boundary condition of no flow through the surface and yields a lift force and pitching moment. The noncirculatory flow model however, does not satisfy the Kutta condition. This is seen by evaluating the pressure difference equation for the noncirculatory flow at the trailing edge, $x=1$. This pressure difference should be zero to satisfy the Kutta condition. Therefore there needs to be another flow included to account for this and assure that the flow will leave the trailing edge in a smooth fashion.

The circulatory flow is developed by using a sheet of vortices on the body as well as a sheet in the wake. The velocity potential for a point vortex is given as[4].

$$
\phi_{\text {vortex }}=\frac{\Gamma}{2 \pi} \tan ^{-1} \frac{Y-Y_{1}}{X-X_{1}}
$$

where, $\Gamma$ is the strength of the vortex.

The wake vortex sheet moves downstream, along the $x$-axis, at the free stream velocity. For a truly correct solution the wake vortex locations are a function of the airfoil motion; but for small disturbances, a flat wake approximation will lead to negligible error. The magnitude of the total shed wake circulation will be equal and opposite to the circulation on the airfoil, and according the Kelvin's circulation theorem, the combined magnitude of the airfoil and wake circulation will be constant for all time[4].

The velocity potential for a pair of point vortices, one outside the circle in the wake at $X_{0}$ and the other of opposite strength at the image position inside the circle, $1 / X_{0}$ is given 
as,

$$
\phi_{\Gamma_{0}}=\frac{\Gamma}{2 \pi}\left[\tan ^{-1}\left(\frac{Y-Y_{0}}{X-X_{0}}\right)-\tan ^{-1}\left(\frac{Y-Y_{0}}{X-\frac{1}{X_{0}}}\right)\right]
$$

where, $\Gamma$ is the strength of each point vortex, and the variables $X$ and $Y$ are spatial variables in the unmapped plane. The velocity perpendicular to the circle due to this vortex pair will be zero when the vortices have the same strength. The equal and opposite strength vortices secures the condition that the total circulation is zero in the domain and the no penetration boundary condition is maintained. Note that these vortices will map to the same location in the mapped plane, but on different Riemann surfaces. Figure 3.4 shows a conformal mapping representation of the vortex pair.

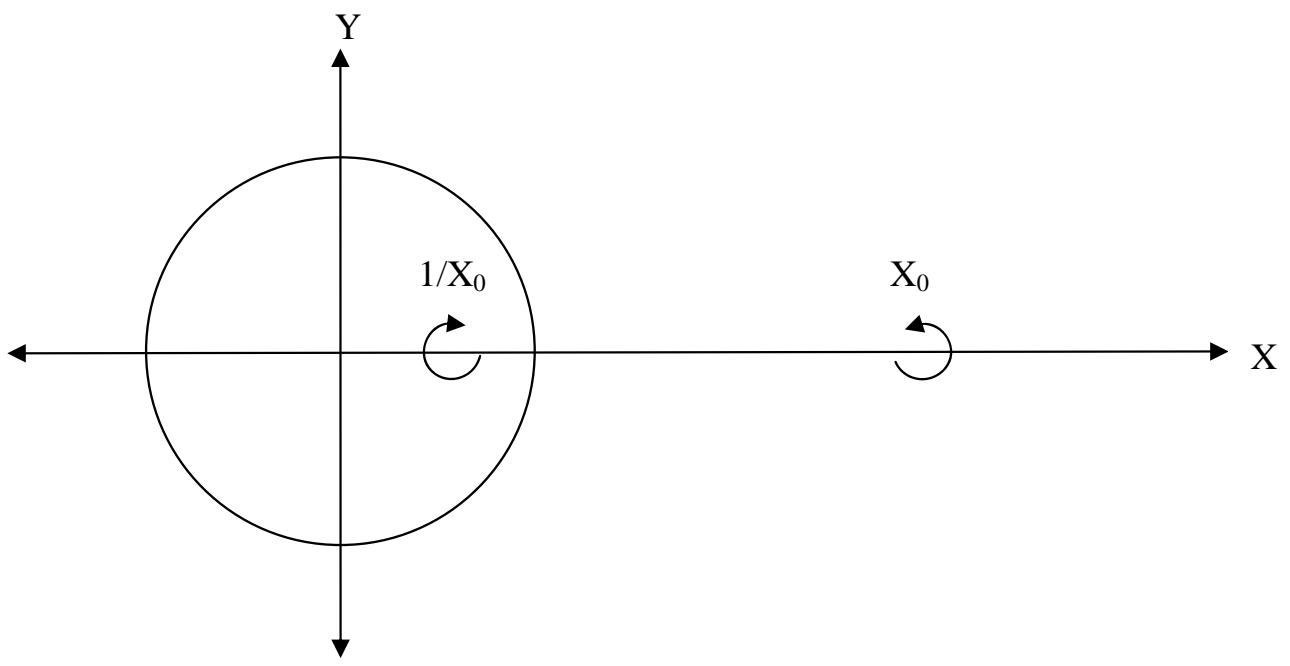

Figure 3.4: Vortex locations

Recalling the Joukowski mapping function, Eq. (3.2), points on the real axis map to another location on the real axis. Therefore the vortex sheet in the mapped plane will be on the real axis as in the unmapped plane. Using properties of tangents, Eq. (3.23) becomes, 


$$
\phi_{\Gamma_{0}}=\frac{\Gamma}{2 \pi} \tan ^{-1}\left[\frac{\left(X-\frac{1}{X_{0}}\right) Y}{X^{2}-\left(X+\frac{1}{X_{0}}\right) X+Y^{2}+1}\right]
$$

Applying the Joukowski mapping, the velocity potential for the vortex pair is given as,

$$
\phi_{\Gamma_{0}}=-\frac{\Gamma}{2 \pi} \tan ^{-1}\left[\frac{\sqrt{1-x^{2}} \sqrt{x_{0}^{2}-1}}{1-x x_{0}}\right]
$$

where, $x$ is the chordwise variable in the mapped plane, and $x_{0}$ is the location of both point vortices in the mapped plane, which was shown by Theodorsen.

The assumption of small disturbances implies that the disturbance flow is small enough to assume the wake propagates at the free stream velocity $u$. This property leads to the following relationship,

$$
\frac{\partial x_{0}}{\partial t}=\frac{u}{b} \rightarrow \frac{\partial \phi}{\partial x_{0}} \frac{\partial x_{0}}{\partial t}=\frac{\partial \phi}{\partial t}=\frac{\partial \phi}{\partial x_{0}} \frac{u}{b}
$$

Combining Eq. (3.26) with the linearized Bernoulli equation, Eq. (3.17), the pressure difference due to the circulatory flow is given as,

$$
\Delta p=-\frac{2 \rho u}{b}\left[\frac{\partial \phi}{\partial x}+\frac{\partial \phi}{\partial x_{0}}\right]
$$

Evaluation of the pressure difference Eq. (3.27) by way of Eq. (3.25) yields pressure difference in terms of circulation, 


$$
\Delta p=-\frac{\rho u b \Gamma}{\pi}\left[\frac{x_{0}+x}{\sqrt{1-x^{2}} \sqrt{x_{0}^{2}-1}}\right]
$$

The lift on the airfoil due to the vortex pair comes from integrating the pressure expression along the chord, and is given as,

$$
\begin{aligned}
& L_{C}=-\frac{\rho u \Gamma}{\pi} \int_{-1}^{1} \frac{x_{0}+x}{\sqrt{1-x^{2}} \sqrt{x_{0}^{2}-1}} d x \\
& L_{C}=-\rho u \Gamma \frac{x_{0}}{\sqrt{x_{0}^{2}-1}}
\end{aligned}
$$

This is a continuous model; therefore the strength of the circulation shed into the wake stretched along the $x$-axis is expressed as,

$$
\Gamma=b \gamma_{w}\left(x_{0}, t\right) d x_{0}
$$

where, $\gamma_{w}\left(x_{0}, t\right)$ is the circulation per unit length. Using this substitution, and integrating the wake vortex sheet from the trailing edge to infinity, yields the circulatory lift,

$$
L_{C}=-\rho u b \int_{1}^{\infty} \frac{x_{0}}{\sqrt{x_{0}^{2}-1}} \gamma_{w}\left(x_{0}, t\right) d x_{0}
$$

The velocity potential for the vortex sheet is given as,

$$
\phi_{\Gamma}=-\frac{b}{2 \pi} \int_{1}^{\infty} \tan ^{-1}\left[\frac{\sqrt{1-x^{2}} \sqrt{x_{0}^{2}-1}}{1-x x_{0}}\right] \gamma_{w}\left(x_{0}, t\right) d x_{0}
$$


The expression for the pitching moment is derived in the same fashion.

$$
\begin{aligned}
& M_{C}=-\frac{\rho u b^{2} \Gamma}{\pi} \int_{-1}^{1} \frac{x_{0}+x}{\sqrt{1-x^{2}} \sqrt{x_{0}^{2}-1}} x d x \\
& M_{C}=-\frac{\rho u b^{2}}{2} \int_{1}^{\infty}\left\{\sqrt{\frac{x_{0}+1}{x_{0}-1}}-\frac{x_{0}}{\sqrt{x_{0}^{2}-1}}\right\} \gamma_{w}\left(x_{0}, t\right) d x_{0}
\end{aligned}
$$

The magnitude of the circulation, $\gamma_{w}\left(x_{0}, t\right)$, is determined by the Kutta condition. The Kutta condition states that the flow must leave the trailing edge smoothly[4]. One way to enforce this is to say that the pressure on the upper surface and lower surface at the trailing edge are equal. Another way is to enforce that there be no infinite velocities at the trailing edge, $x=1$.

$$
\frac{\partial}{\partial x}\left(\phi_{\Gamma}+\phi_{\alpha}+\phi_{\dot{h}}+\phi_{\dot{\alpha}}+\phi_{\kappa}+\phi_{\dot{\kappa}}+\ldots+\phi_{\kappa_{N}}+\phi_{\dot{\kappa}_{N}}\right) \neq \infty
$$

The terms in this equation are the disturbance velocities due to each deformation shape and the circulation. The equations for the velocity potentials of each deformation shape and the circulation are differentiated and evaluated at $x=1$, the trailing edge. Stating that the velocity be finite at the trailing edge results in a very useful equation for the first five deformation shape.

$$
\begin{aligned}
\frac{1}{2 \pi} \int_{1}^{\infty} \sqrt{\frac{x_{0}+1}{x_{0}-1}} \gamma_{w}\left(x_{0}, t\right) d x_{0} & =u \alpha+\dot{h}+\frac{b}{2} \dot{\alpha}+\frac{u b}{2} \kappa \\
& +\frac{u b^{2}}{8} \kappa_{2}+\frac{u b^{3}}{48} \kappa_{3}=Q
\end{aligned}
$$

This result is quite useful because the integral on the right hand side is shown to be equal to a function of the airfoil motion, denoted as Q[1]. Eq. (3.35) is the exact same result 
obtained by Theodorsen for the pitch and plunge terms. However, the deformation terms are an extension to Theodorsen theory. Multiplying Eq. (3.31) by $Q$ and dividing by the integral on the right hand side of Eq. (3.35), the circulatory lift becomes,

$$
L_{C}=-2 \pi \rho u b Q \frac{\int_{1}^{\infty} \frac{x_{0}}{\sqrt{x_{0}^{2}-1}} \gamma_{w}\left(x_{0}, t\right) d x_{0}}{\int_{1}^{\infty} \sqrt{\frac{x_{0}+1}{x_{0}-1}} \gamma_{w}\left(x_{0}, t\right) d x_{0}}
$$

which was shown by Theodorsen. The significance of the ratio of integrals is discussed in detail further in the following section. The circulatory pitching moment is derived in the same fashion as shown by Theodorsen[1].

$$
M_{C}=-\pi \rho u b^{2} Q\left[\frac{\int_{1}^{\infty} \frac{x_{0}}{\sqrt{x_{0}^{2}-1}} \gamma_{w}\left(x_{0}, t\right) d x_{0}}{\int_{1}^{\infty} \sqrt{\frac{x_{0}+1}{x_{0}-1}} \gamma_{w}\left(x_{0}, t\right) d x_{0}}-1\right]
$$

The circulatory lift and pitching moment equations are the exact same as Theodorsen derived. The only thing which makes this different is the airfoil motion term, $Q$, which contains camber terms which Theodorsen did not have.

\subsubsection{Theodorsen's Function}

Thus far there has been no mention of the time dependence of the motion other than it must fit into a small disturbances assumption. Restricting the motion to be harmonic allows for a solution to the ratio of integrals in Eqs. (3.36) and (3.37). The motion is written as a function of frequency of oscillation by restricting the flow to simple harmonic motion in the following form. 


$$
\begin{aligned}
\alpha(x, t) & =\bar{\alpha} e^{i\left(\omega t+\varphi_{\alpha}\right)} \\
h(x, t) & =\bar{h} e^{i\left(\omega t+\varphi_{h}\right)} \\
\kappa(x, t) & =\bar{\kappa} e^{i\left(\omega t+\varphi_{\kappa}\right)}
\end{aligned}
$$

where, $\bar{\alpha}, \bar{h}$ and $\bar{\kappa}$ are magnitudes of oscillation. The terms $\varphi_{\alpha}, \varphi_{h}$ and $\varphi_{\kappa}$ are the phase's of the motion. The frequency is denoted as $\omega$.

The wake vortex sheet strength is also oscillatory, and is written in terms of the reduced frequency, $k=\frac{\omega b}{u}$.

$$
\gamma_{w}(x, t)=\bar{\gamma}_{w} e^{i \omega\left(t-\frac{b x}{u}\right)}=\bar{\gamma}_{w} e^{i(\omega t-k x)}
$$

where, $\bar{\gamma}_{w}$ is the magnitude of oscillation of the circulation.

The ratio of integrals in the circulatory loading expressions is denoted $C$, which was shown by Theodorsen to be purely a function of the reduced frequency, $k$.

$$
\begin{aligned}
C & =\frac{\int_{1}^{\infty} \frac{x_{0}}{\sqrt{x_{0}^{2}-1}} \gamma_{w}\left(x_{0}, t\right) d x_{0}}{\int_{1}^{\infty} \sqrt{\frac{x_{0}+1}{x_{0}-1}} \gamma_{w}\left(x_{0}, t\right) d x_{0}}=\frac{\bar{\gamma}_{w} e^{i \omega t} b \int_{1}^{\infty} \frac{x_{0}}{\sqrt{x_{0}^{2}-1}} e^{-i k x_{0}} d x_{0}}{\bar{\gamma}_{w} e^{i \omega t} b \int_{1}^{\infty} \sqrt{\frac{x_{0}+1}{x_{0}-1}} e^{-i k x_{0}} d x_{0}} \\
= & \frac{\int_{1}^{\infty} \frac{x_{0}}{\sqrt{x_{0}^{2}-1}} e^{-i k x_{0}} d x_{0}}{\int_{1}^{\infty} \sqrt{\frac{x_{0}+1}{x_{0}-1}} e^{-i k x_{0}} d x_{0}}=C(k)
\end{aligned}
$$

This ratio of integrals over $x_{0}$ is only a function of the reduced frequency, and take the form of Hankel functions[23].

$$
C(k)=F(k)+i G(k)=\frac{H_{1}^{(2)}(k)}{H_{1}^{(2)}(k)+i H_{0}^{(2)}(k)}
$$


where the Hankel functions in Eq. (3.41) are defined by a complex combination of Bessel functions of the first and second kind in the form of,

$$
H_{n}^{(2)}(k)=J_{n}(k)-i Y_{n}(k)
$$

Details of the solution to Theodorsen's function are given in Appendix A.

A complex plot of Theodorsen's function from reduced frequency values of zero to infinity is shown in Figure 3.5. The function is 1 when the reduced frequency is zero and 0.5 when the reduced frequency is infinity. It reduces the amplitude of the circulatory lift with increasing reduced frequency of oscillation. It also shifts the phase of the lift relative to the motion.

Figure 3.5 gives much insight into the meaning of Theodorsen's function. Essentially, $C(k)$ is a lift reduction factor as a function of the frequency of oscillation. Perhaps a very important note is that this plot shows a value of reduced frequency where it could be sufficient to assume a quasi-steady flow and ignore the effect of the wake and the higher order terms. For values of $0<k<0.025$, the flow could be assumed quasi-steady. Theodorsen's function being only a function of the reduced frequency in itself is a significant piece of information. This shows that the reduced frequency is essentially the measure of the unsteadiness in the flow, and therefore a flow with equal unsteadiness can be achieved with different combinations of the frequency, chord length and free-stream velocity, as long as these combine to the same value of reduced frequency.

\subsubsection{Total Lift and Pitching Moment Expressions}

The complete lift and pitching moment expressions are simply the addition of the circulatory and noncirculatory components. The final lift expression is complex where the amplitude of the complex number is the amplitude of oscillation, and the phase is the phase of the force and moment relative to the phase of the motion. Using the harmonic motion of Eq. (3.38), 


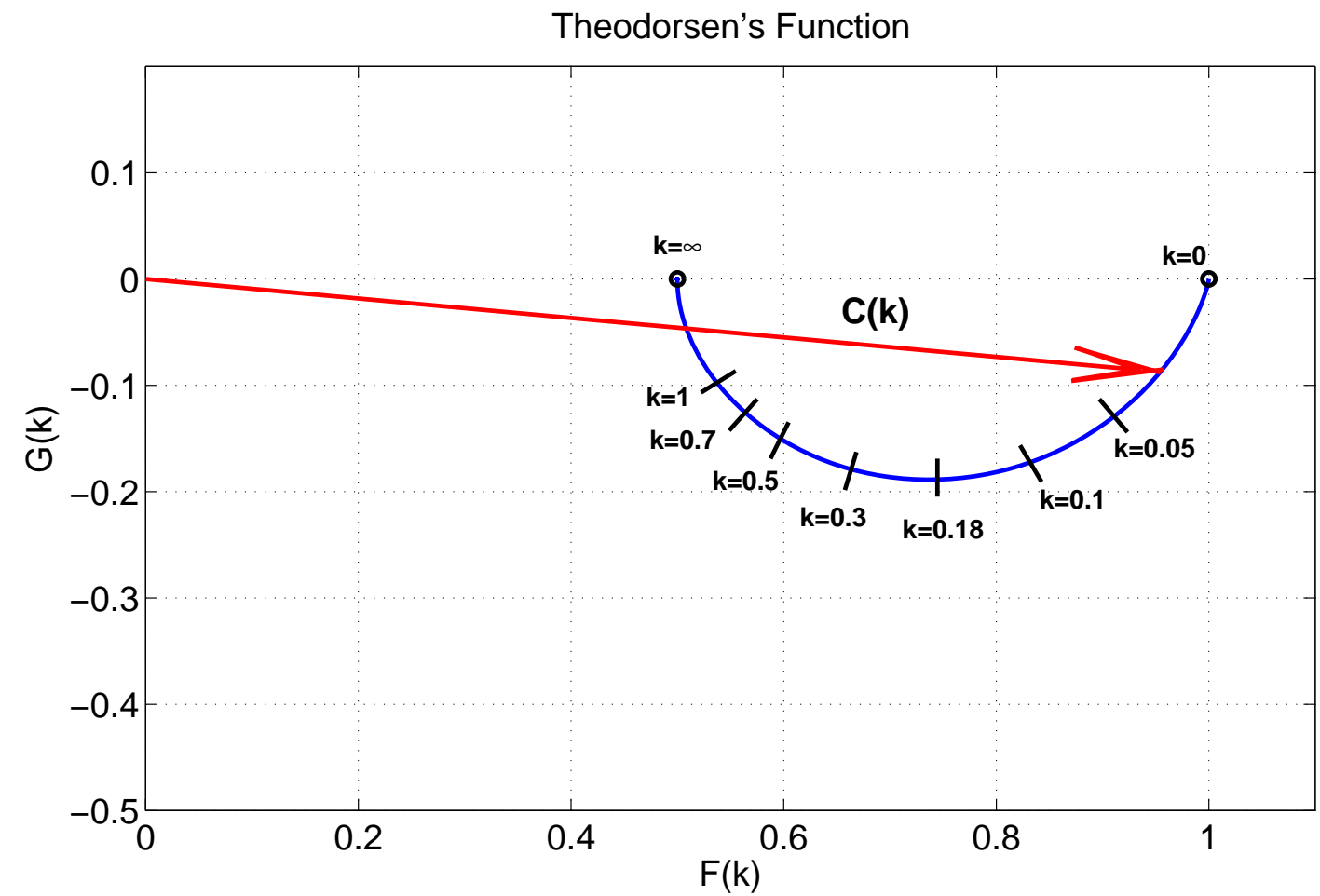

Figure 3.5: Theodorsen's function

the different airfoil motions can have different phases relative to each other.

Tables 3.2 and 3.3 represent the lift and pitching moment contributions from each shape. These tables give the airloads due to each deformation shape, corresponding velocities and corresponding acceleration. The first two rows of each table match Theodorsen. The top line represents the time derivative of the terms in the left column. The terms in the table are multiplied by the respective derivative of the respective deformation variable. The lift force components of Table 3.2 easily show which airfoil motion shapes contribute to the lift. Moreover, the existence of Theodorsen's function in the terms describes whether the lift force is due to circulatory or noncirculatory flow. The static airfoil shapes, the first column 
Table 3.2: Lift force components

\begin{tabular}{cccc}
\hline \hline & 1 & $\frac{\partial}{\partial t}$ & $\frac{\partial^{2}}{\partial t^{2}}$ \\
\hline $\mathrm{h}$ & 0 & $-2 \pi \rho u b C(k)$ & $-\pi \rho b^{2}$ \\
$\alpha$ & $-2 \pi \rho u^{2} b C(k)$ & $-\pi \rho u b^{2}(C(k)+1)$ & 0 \\
$\kappa$ & $-\pi \rho u^{2} b^{2} C(k)$ & 0 & $-\frac{1}{8} \pi \rho b^{4}$ \\
$\kappa_{2}$ & $-\frac{1}{4} \pi \rho u^{2} b^{3} C(k)$ & 0 & 0 \\
$\kappa_{3}$ & $-\frac{1}{24} \pi \rho u^{2} b^{4} C(k)$ & 0 & 0 \\
\hline
\end{tabular}

Table 3.3: Pitching moment components

\begin{tabular}{cccc}
\hline \hline & 1 & $\frac{\partial}{\partial t}$ & $\frac{\partial^{2}}{\partial t^{2}}$ \\
\hline $\mathrm{h}$ & 0 & $-\pi \rho u b^{2} C(k)$ & 0 \\
$\alpha$ & $\pi \rho u^{2} b^{2} C(k)$ & $\frac{1}{2} \pi \rho u b^{3}(C(k)-1)$ & $-\frac{1}{8} \pi \rho b^{4}$ \\
$\kappa$ & $\frac{1}{2} \pi \rho u^{2} b^{3}(C(k)-1)$ & $-\frac{1}{4} \pi \rho u b^{4}$ & 0 \\
$\kappa_{2}$ & $\frac{1}{8} \pi \rho u^{2} b^{4}(C(k)-1)$ & 0 & $\frac{1}{192} \pi \rho b^{6}$ \\
$\kappa_{3}$ & $\frac{1}{48} \pi \rho u^{2} b^{5}(C(k)-1)$ & 0 & 0 \\
\hline
\end{tabular}

of Table 3.2, are only circulatory forces, which is expected because there are no accelerations to contribute to the noncirculatory flow. The plunge displacement has no effect on the lift. However, the second column shows the plunge velocity contributes to lift in the same form as a static angle of attack. This is obvious because a constant plunge velocity is analogous to a static angle of attack. The pitch velocity also contributes a circulatory lift term, but also a noncirculatory lift term. The third column shows that only the plunge and quadratic camber accelerations lead to a lift, and this term is noncirculatory.

The pitching moment components of Table 3.3 show the same type of results as the lift. It is not quite as easy to see the difference between circulatory and noncirculatory terms because the pitching moment terms due to circulation are not always multiplied by $C(k)$, as shown in Eq. (3.37). The pitching moment terms due to circulation are multiplied by $C(k)-1$, making it still easy to see which airfoil shapes contribute to noncirculatory and circulatory flow. As expected, the pitching moment is zero for plunge displacement. The rest of the static shapes lead to pitching moment due to the circulation. As with the lift, the plunge velocity resembles the static angle of attack term and the pitch rate term is circulatory. The quadratic camber velocity leads to a noncirculatory lift term because the 
changing shape results in local accelerations. Finally, the acceleration of the shapes leads to noncirculatory terms.

There are almost no dynamic terms for the deformations. Most of the unsteady terms come from the rigid body motion. The results of this derivation use Chebychev polynomials, however, if a different set of orthogonal functions was used, these results would be different, perhaps including more dynamic terms for the deformations.

\subsection{Thrust Derivation}

Force in ideal flow involves only nonviscous components, which for steady flow results in only a lift force perpendicular to the direction of flow. Unsteady flows however can have force in the horizontal direction parallel to the direction of flow. Depending on the airfoil motion, this force can be either positive, which is a thrust, or negative, which is a drag. The following derivation extends the method used by Garrick[2] to calculate the horizontal force for the unsteady motion described by Eq. (3.6).

The force in the horizontal direction consists of two components, the component of the pressure which acts in the direction of the flow, and the force due to leading edge suction. The force due to leading edge suction will always be positive, as will be shown in this section, and the component of the pressure in the flow direction can be either sign. The derivation will be done assuming positive force in the negative $x$ direction, which is the direction of positive thrust.

\subsubsection{Thrust due to Pressure}

The component of the thrust due to the lift force is simply the net force multiplied by the local slope. This expression is integrated along the chord to yield the thrust force due to a general airfoil shape. 


$$
T_{L}=b \int_{-1}^{1} \Delta p \frac{\partial y}{\partial x} d x
$$

\subsubsection{Thrust due to Leading Edge Suction}

The leading edge suction velocity comes from the vorticity at the leading edge. Referring to Eq. (3.34), the velocity at the trailing edge $x=1$ becomes finite. The leading edge suction velocity is derived from the same equation, but at the leading edge, where $x=-1$. Upon evaluating this equation at the leading edge, the velocity becomes infinite. This is mathematically correct, but of course not physically possible. This airloads theory assumes a thin airfoil, which is a flat plate. A true airfoil would have thickness associated with it, and when the airfoil is at some angle of attack the stagnation point moves away from the leading edge, $x=-1$ to some other location, as shown in Figure 3.6. The flow stops at the stagnation point and must travel around the airfoil's leading edge toward the other surface and continue on. As the airfoil thickness decreases to zero, the leading edge radius also approaches zero requiring the flow to turn exactly $180^{\circ}$ at the exact point in the leading edge, $x=-1$. This turn requires an infinite acceleration, and therefore an infinite velocity. The true leading edge suction can be found by knowing the order of the infinity.

The leading edge suction goes to infinity in a functional form given by $1 / \sqrt{1+x}$ as shown by von Kármán and Burgers[3]. Using this, the vorticity expression for the leading edge is expressed as a function of the leading edge suction velocity.

$$
2 \frac{\partial}{\partial x}\left(\phi_{\Gamma}+\phi_{\alpha}+\phi_{\dot{h}}+\phi_{\dot{\alpha}}+\ldots+\phi_{\kappa_{N}}+\phi_{\dot{\kappa}_{N}}\right)_{x=-1}=\frac{2 S}{\sqrt{1+x}}
$$

where $S$ is the leading edge suction as shown by Garrick[2].

The derivative of the circulatory velocity potential term has an integral to infinity in it which is the denominator of Theodorsen's function. Using this and Theodorsen's function, 


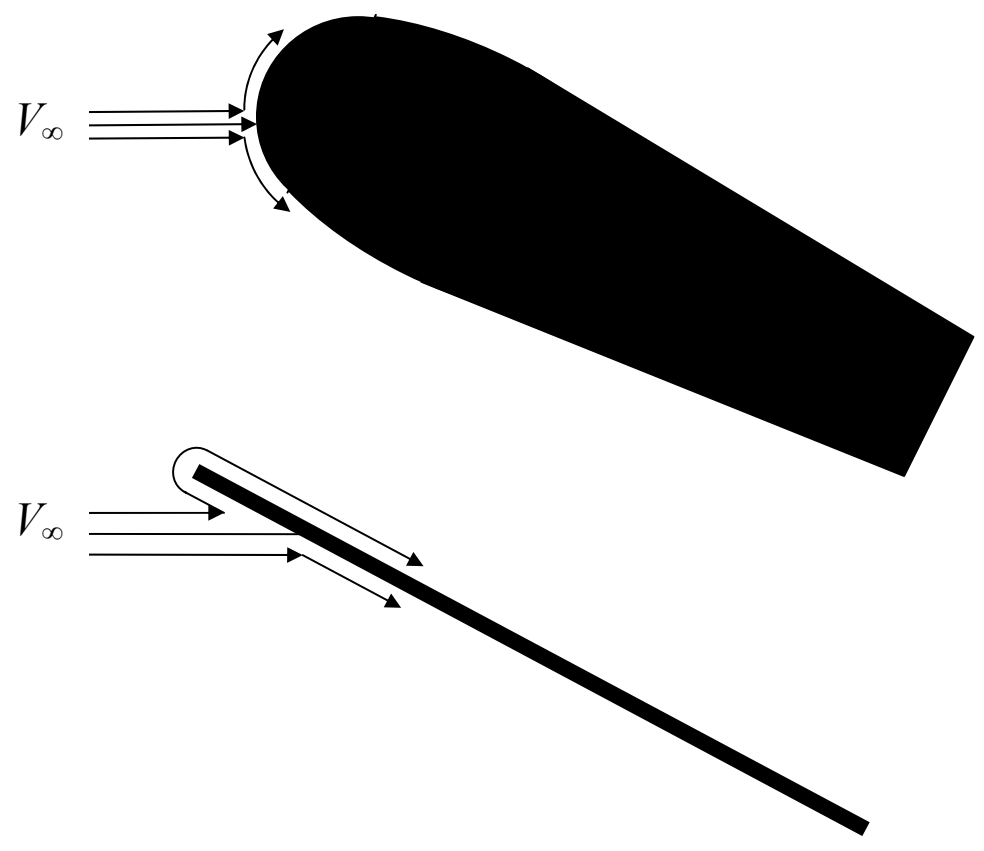

Figure 3.6: Fluid flow around leading edge

along with Eq. (3.35), an expression for $S$ is derived,

$$
S=\frac{\sqrt{2}}{2}(2 C(k) Q-Q)+\left[\frac{\partial}{\partial x}\left(\phi_{\alpha}+\phi_{\dot{h}}+\phi_{\dot{\alpha}}+\ldots+\phi_{\kappa_{N}}+\phi_{\dot{\kappa}_{N}}\right) \frac{\sqrt{1+x}}{b}\right]_{x=-1}
$$

The resulting leading edge suction velocity due to the first five airfoil displacement /deformation modes are given in Table 3.4. The force due to this velocity is simply $\pi \rho b S^{2}[4]$. The leading edge suction velocities for the pitch and plunge terms match Garrick[2].

Table 3.4: Leading edge suction velocities

\begin{tabular}{cccc}
\hline \hline & 1 & $\frac{\partial}{\partial t}$ & $\frac{\partial^{2}}{\partial t^{2}}$ \\
\hline $\mathrm{h}$ & 0 & $\sqrt{2} C(k)$ & 0 \\
$\alpha$ & $\sqrt{2} C(k) u$ & $\frac{\sqrt{2}}{2} b(C(k)-1)$ & 0 \\
$\kappa$ & $\frac{\sqrt{2}}{2} b u(C(k)-1)$ & 0 & 0 \\
$\kappa_{2}$ & $\frac{\sqrt{2}}{8} b^{2} u C(k)$ & 0 & 0 \\
$\kappa_{3}$ & $\frac{\sqrt{2}}{48} b^{3} u(C(k)-1)$ & 0 & 0 \\
\hline
\end{tabular}




\subsubsection{Total Thrust Force}

The thrust force due to both the lift component and the leading edge suction component combine to result in Eq. (3.46). The squared term shows that drag will contain many squared and cross coupling terms; and therefore the Tables following show each component of the thrust up through the first five airfoil deformations. These tables are interpreted by multiplying the terms in the table by the corresponding terms in the top row and left column.

$$
T=T_{L E S}+T_{L}=\pi \rho b S^{2}+b \int_{-1}^{1} \Delta p \frac{\partial y}{\partial x} d x
$$

Table 3.5: Thrust force due to $\mathrm{h}$ and $\alpha$

\begin{tabular}{ccccc}
\hline \hline & $\dot{h}$ & $\ddot{h}$ & $\alpha$ & $\dot{\alpha}$ \\
\hline$\dot{h}$ & $2 \pi \rho b C(k)^{2}$ & 0 & $2 \pi \rho u b\left(2 C(k)^{2}-C(k)\right)$ & $2 \pi \rho b^{2}\left(C(k)^{2}-C(k)\right)$ \\
$\ddot{h}$ & - & 0 & $-\pi \rho b^{2}$ & 0 \\
$\alpha$ & - & - & $2 \pi \rho u^{2} b\left(C(k)^{2}-C(k)\right)$ & $\pi \rho u b^{2}\left(2 C(k)^{2}-3 C(k)-1\right)$ \\
$\dot{\alpha}$ & - & - & - & $\frac{1}{2} \pi \rho b^{3}\left(C(k)^{2}-2 C(k)-1\right)$ \\
\hline
\end{tabular}

The resulting expressions in Table 3.5 match the solution of Garrick [2]. There are no thrust terms with plunge displacement in them, which is expected because a static flat plate at no angle of attack will not produce any forces. The thrust force for pitching and plunging motions is almost entirely dependent on circulatory terms. The plunge acceleration at an

Table 3.6: Thrust force due to $\kappa$

\begin{tabular}{cccc}
\hline \hline & $\kappa$ & $\dot{\kappa}$ & $\ddot{\kappa}$ \\
\hline$\dot{h}$ & $\pi \rho b^{2}\left(C(k)^{2}-C(k)\right)$ & 0 & 0 \\
$\ddot{h}$ & 0 & 0 & 0 \\
$\alpha$ & $2 \pi \rho u^{2} b^{2}\left(C(k)^{2}-C(k)\right)$ & 0 & $\frac{1}{8} \pi \rho b^{4}$ \\
$\dot{\alpha}$ & $\frac{1}{2} \pi \rho b^{3}\left(2 C(k)^{2}-C(k)+1\right)$ & 0 & 0 \\
$\ddot{\alpha}$ & $-\frac{1}{8} \pi \rho b^{4}$ & 0 & 0 \\
$\kappa$ & $\frac{1}{2} \pi \rho u^{2} b^{3}\left(C(k)^{2}-C(k)\right)$ & $-\frac{1}{4} \pi \rho v b^{4}$ & 0 \\
$\dot{\kappa}$ & - & 0 & 0 \\
$\ddot{\kappa}$ & - & - & 0 \\
\hline
\end{tabular}


Table 3.7: Thrust force due to $\kappa_{2}$

\begin{tabular}{cccc}
\hline \hline & $\kappa_{2}$ & $\dot{\kappa}_{2}$ & $\ddot{\kappa}_{2}$ \\
\hline$\dot{h}$ & $\frac{1}{4} \pi \rho u b^{3}\left(2 C(k)^{2}-C(k)\right)$ & 0 & 0 \\
$\ddot{h}$ & 0 & 0 & 0 \\
$\alpha$ & $\frac{1}{2} \pi \rho u^{2} b^{3}\left(C(k)^{2}-C(k)\right)$ & 0 & 0 \\
$\dot{\alpha}$ & $\frac{1}{8} \pi \rho u b^{4}\left(2 C(k)^{2}-3 C(k)+1\right)$ & 0 & 0 \\
$\ddot{\alpha}$ & 0 & 0 & 0 \\
$\kappa$ & $\frac{1}{4} \pi \rho u^{2} b^{4}\left(C(k)^{2}-C(k)\right)$ & $\frac{1}{192} \pi \rho b^{6}$ & 0 \\
$\dot{\kappa}$ & 0 & 0 & 0 \\
$\ddot{\kappa}$ & $-\frac{1}{192} \pi \rho b^{6}$ & 0 & 0 \\
$\kappa_{2}$ & $\frac{1}{32} \pi \rho u^{2} b^{5}\left(C(k)^{2}-C(k)\right)$ & $-\frac{1}{96} \pi \rho u b^{6}$ & 0 \\
$\dot{\kappa}_{2}$ & - & 0 & 0 \\
$\ddot{\kappa}_{2}$ & - & - & 0 \\
\hline
\end{tabular}

Table 3.8: Thrust force due to $\kappa_{3}$

\begin{tabular}{cccc}
\hline \hline & $\kappa_{3}$ & $\dot{\kappa}_{3}$ & $\ddot{\kappa}_{3}$ \\
\hline$\dot{h}$ & $\frac{1}{24} \pi \rho u b^{4}\left(2 C(k)^{2}-C(k)\right)$ & 0 & 0 \\
$\ddot{h}$ & 0 & 0 & 0 \\
$\alpha$ & $\frac{1}{12} \pi \rho u^{2} b^{4}\left(C(k)^{2}-C(k)\right)$ & 0 & 0 \\
$\dot{\alpha}$ & 0 & 0 & 0 \\
$\ddot{\alpha}$ & $\frac{1}{48} \pi \rho u b^{5}\left(2 C(k)^{2}-3 C(k)-1\right)$ & 0 & 0 \\
$\kappa$ & $\frac{1}{24} \pi \rho u^{2} b^{5}\left(C(k)^{2}-C(k)\right)$ & 0 & 0 \\
$\dot{\kappa}$ & 0 & 0 & 0 \\
$\ddot{\kappa}$ & 0 & 0 & 0 \\
$\kappa_{2}$ & $\frac{1}{96} \pi \rho u^{2} b^{6}\left(C(k)^{2}-C(k)\right)$ & 0 & $\frac{1}{9216} \pi \rho b^{8}$ \\
$\dot{\kappa}_{2}$ & 0 & 0 & 0 \\
$\ddot{\kappa}_{2}$ & $-\frac{1}{9216} \pi \rho b^{8}$ & 0 & 0 \\
$\kappa_{3}$ & $\frac{1}{1152} \pi \rho u^{2} b^{7}\left(C(k)^{2}-C(k)\right)$ & $-\frac{1}{4608} \pi \rho u b^{8}$ & 0 \\
$\dot{\kappa}_{3}$ & - & 0 & 0 \\
$\ddot{\kappa}_{3}$ & - & - & 0 \\
\hline
\end{tabular}


angle of attack results in a thrust term which is noncirculatory.

The camber terms of Tables 3.6 through 3.8 result in thrust terms, but mostly only with the static shape multiplied by plunge and pitch terms. There are several terms including camber rate and camber acceleration for the three different camber shapes listed, and they are noncirculatory. 


\section{Chapter 4}

\section{Verification of Aerodynamic Theory}

The theory developed in the previous chapter is an extension of unsteady aerodynamics theories developed by Theodorsen and Garrick. This theory is based upon the solution to Laplace's equation for simple flows, the velocity potential approach, the no penetration boundary condition on the airfoil surface, and the Kutta condition. This solution assumes that the unsteady motion is harmonic. The lift, pitching moment, and thrust derived in Chapter 3 match the solutions of Theodorsen and Garrick exactly for the rigid body motion terms. However, the theory in Chapter 3 is not the only deformable airloads theory; a similar theory has been developed by Peters. Peters' theory is a general theory for any arbitrary motion that requires the use of Chebychev polynomials to define the deformation shapes[17]. Peters has shown for the pitch and plunge cases, assuming the motion to be harmonic and the wake to be flat, that Theodorsen theory can be recovered from his formulation. It follows Peter's theory will reduce to the extension to Theodorsen and Garrick theory derived in this work.

\subsection{Peters' Unsteady Airloads Theory}

The unsteady airloads theory developed by Peters is described briefly in this section in order to show the equations which will be used in comparison to the theory in this work. Peters 
expresses the no penetration boundary condition as,

$$
\begin{aligned}
w & =\bar{v}+\lambda \\
& =u_{0} \frac{\partial h}{\partial x}+\frac{\partial h}{\partial t}+v_{0}+v_{1} \frac{x}{b}
\end{aligned}
$$

where $w$ is the total induced velocity, $\bar{v}$ is the velocity due to the bound vorticity, and $\lambda$ is the velocity due to the wake vorticity. The total induced velocity is a function of the airfoil shape and motion, as shown in the second half of the equation. The airfoil shape is given by $h(x, t)$, and so the derivatives $\partial h / \partial x$ and $\partial h / \partial t$ are the local slope of the airfoil and local vertical velocity of the airfoil as a function of the chordwise location $x$. The horizontal and vertical free stream velocities are $u_{0}$ and $v_{0}$ respectively. The term $v_{1}$ is the velocity gradient and $b$ is the semichord.

The velocity $\bar{v}$ is expressed as the distribution of vortices on the airfoil is given by $\gamma_{b}(\xi, t)[17]$.

$$
\bar{v}=-\frac{1}{2 \pi} \int_{-b}^{b} \frac{\gamma_{b}(\xi, t)}{x-\xi} d \xi
$$

The pressure difference across the airfoil is expressed for the body[17],

$$
\Delta P=\rho u_{0} \gamma_{b}+\rho \int_{-b}^{x} \frac{\partial \gamma_{b}}{\partial t} d \xi \quad(-b<x<b)
$$

as well as for the wake[17],

$$
0=u_{0} \gamma_{w}+\frac{\partial \Gamma}{\partial t}+\rho \int_{b}^{x} \frac{\partial \gamma_{w}}{\partial t} d \xi \quad(b<x)
$$


Therefore applying the boundary condition of no penetration results in,

$$
\frac{\partial \lambda}{\partial t}+u_{0} \frac{\partial \lambda}{\partial x}=\frac{1}{2 \pi} \frac{\frac{d \Gamma}{d t}}{b-x}
$$

where $\Gamma$ is circulation[17].

These equations are solved using the Glauert change variable $x=b \cos \varphi$. The expressions for $\bar{v}, h$ and $\lambda$ become,

$$
\begin{array}{r}
\bar{v}=\sum_{n=0}^{\infty} \gamma_{n} \cos (n \varphi) \\
\lambda=\sum_{n=0}^{\infty} \lambda_{n} \cos (n \varphi) \\
h(x)=\sum_{n=0}^{\infty} h_{n} \cos (n \varphi)
\end{array}
$$

Using Eq. (4.6), the pressure difference is integrated along the chord yield the lift[17].

$$
L=-2 \pi \rho u_{0} b\left(w_{0}-\lambda_{0}\right)-\pi \rho u_{0} b w_{1}-\pi \rho b^{2}\left(\dot{w}_{0}-\frac{1}{2} \dot{w}_{2}\right)
$$

The pitching moment is found by integrating the pressure difference multiplied by the moment arm $x[17]$.

$$
M=\pi \rho u_{0} b\left(w_{0}-\lambda_{0}\right)-\frac{1}{2} \pi \rho u_{0} b w_{2}-\frac{1}{8} \pi \rho b^{2}\left(\dot{w}_{1}-\dot{w}_{3}\right)
$$

Finally, the drag expression is given as[17], 


$$
\begin{aligned}
D & =-2 \pi \rho b\left(\dot{h}_{0}-\lambda_{0}\right)\left[\left(\dot{h}_{0}-\lambda_{0}\right)+u_{0} \sum_{n=1}^{\infty} \frac{n h_{n}}{b}\right] \\
& +2 \pi \rho \sum_{n=1}^{\infty} h_{n}\left[\frac{b}{4}\left(\ddot{h}_{n-1}-\ddot{h}_{n+1}\right)+n u_{0} \dot{h}_{n}\right]+\pi \rho \frac{b}{2} h_{1} \ddot{h}_{0}
\end{aligned}
$$

The w's result from Eq. (4.1)[17],

$$
\begin{aligned}
& w_{0}=\dot{h}_{0}+u_{0} \sum_{n=1,3,5}^{\infty} \frac{n h_{n}}{b} \\
& w_{1}=\dot{h}_{1}+2 u_{0} \sum_{n=2,4,6}^{\infty} \frac{n h_{n}}{b} \\
& w_{m}=\dot{h}_{m}+2 u_{0} \sum_{n=m+1, m+3}^{\infty} \frac{n h_{n}}{b} \quad m \geq 2
\end{aligned}
$$

\subsection{Application of Peters' Theory to Current Problem}

Peters' formulation is developed for arbitrary airfoil motion. By restricting the motion to be harmonic, and restricting rigid body motion to be small, Theodorsen and Garrick results can be obtained from Peters' theory. Peters showed the relationship between Theodorsen's function, the wake term and the w's used in his derivation.

$$
\lambda_{0}=\left(w_{0}+w_{1}\right)(1-C(k))
$$

Evaluating Peters' theory for the first five orthogonal airfoil shapes, the lift and pitching moment are found in Tables 4.1 and 4.2 .

The airfoil motion used by Peters, Eq. (4.1), is in terms of $h(x)$, which is given in Eq. (4.6). Noting that the $\cos n \varphi$ is equivalent to the Chebychev polynomials, Eq. (3.4), evaluated at $x / b$, the $h_{n}$ 's are equivalent to the $A_{n}$ 's of Eq. (3.6) multiplied by the semichord 
Table 4.1: Lift force components

\begin{tabular}{cccc}
\hline \hline & 1 & $\frac{\partial}{\partial t}$ & $\frac{\partial^{2}}{\partial t^{2}}$ \\
\hline$h_{0}$ & 0 & $-2 \pi \rho u_{0} b C(k)$ & $-\pi \rho b^{2}$ \\
$h_{1}$ & $-2 \pi \rho u_{0}^{2} C(k)$ & $-\pi \rho u_{0} b(C(k)+1)$ & 0 \\
$h_{2}$ & $-4 \pi \rho u_{0}^{2} C(k)$ & 0 & $-\frac{1}{2} \pi \rho b^{2}$ \\
$h_{3}$ & $-6 \pi \rho u_{0}^{2} C(k)$ & 0 & 0 \\
$h_{4}$ & $-8 \pi \rho u_{0}^{2} C(k)$ & 0 & 0 \\
\hline
\end{tabular}

Table 4.2: Pitching moment components

\begin{tabular}{cccc}
\hline \hline & 1 & $\frac{\partial}{\partial t}$ & $\frac{\partial^{2}}{\partial t^{2}}$ \\
\hline$h_{0}$ & 0 & $-\pi \rho u_{0} b^{2} C(k)$ & 0 \\
$h_{1}$ & $\pi \rho u_{0}^{2} b C(k)$ & $\frac{1}{2} \pi \rho u_{0} b^{2}(C(k)-1)$ & $-\frac{1}{8} \pi \rho b^{3}$ \\
$h_{2}$ & $2 \pi \rho u_{0}^{2} b(C(k)-1)$ & $-\pi \rho u_{0} b^{2}$ & 0 \\
$h_{3}$ & $3 \pi \rho u_{0}^{2} b(C(k)-1)$ & 0 & $\frac{1}{8} \pi \rho b^{3}$ \\
$h_{4}$ & $4 \pi \rho u_{0}^{2} b(C(k)-1)$ & 0 & 0 \\
\hline
\end{tabular}

b. The free stream velocity $u$ is $u_{0}$ in Peters' theory. Upon inspecting Tables 4.1 and 4.2 , and comparing to Tables 3.2 and 3.3, it is seen that both the formulations yield the same result. The drag results have the same outcome except for the sign. This is because Peters' sign convention is for the positive force to be in the drag direction, while the extended Garrick theory assumes the force positive in the thrust direction.

The solution for Peters' theory relies on the use of the Glauert change variable, which are the Chebychev polynomials at $x / b$. This is the reason these polynomials were chosen. The results developed in this thesis are essentially Peters' theory for an flat wake with harmonic motion. However, the extended Theodorsen and Garrick theory derived can be formulated for any set of orthogonal functions, such as the Legendre polynomials. This allows many different shapes to be used instead of just Chebychev polynomials. 


\section{Chapter 5}

\section{Case Study}

This chapter is focused on presenting various case studies based on the results of the airloads derivation. Expressions for the amplitude of oscillation and phase of lift and pitching moment relative to the motion is derived. A detailed study into the thrust and how the pressure and leading edge suction components contribute to the thrust for each case, and combinations of them is shown.

\subsection{Lift and Pitching Moment Results}

The lift and pitching moment, Tables 3.2 and 3.3, can easily be made a function of the reduced frequency using the substitution of Eq. (3.38). Using this substitution, the expressions for the lift and pitching moment will be expressed as,

$$
\begin{aligned}
& L=\bar{L} e^{i\left(\omega t+\varphi_{L}\right)}=\bar{L} e^{i \omega t} e^{i \varphi_{L}} \\
& M=\bar{M} e^{i\left(\omega t+\varphi_{M}\right)}=\bar{M} e^{i \omega t} e^{i \varphi_{M}}
\end{aligned}
$$

where, $\bar{L}$ and $\bar{M}$ are the magnitudes of oscillation of the lift and pitching moment respectively; and the $\varphi_{L}$ and $\varphi_{M}$ are the phase relative to the motion for lift and pitching moment respectively. The time dependent term easily cancels from both sides of the equations. 
Therefore the result yields an equation for the lift and pitching moment amplitudes and phases relative to the airfoil motion. The coefficient expressions for the lift and pitching moment amplitude and phase are given in Table 5.1. The lift and pitching moment terms due to each shape can be found by multiplying the term by the left column.

Table 5.1: Lift and pitching moment amplitude and phase terms

\begin{tabular}{ccc}
\hline \hline & $\bar{L} e^{i \varphi_{L}}$ & $\bar{M} e^{i \varphi_{M}}$ \\
\hline$h e^{i \varphi_{h}}$ & $\pi \rho u^{2}\left(k^{2}-i 2 k C(k)\right)$ & $i \pi \rho u^{2} k C(k)$ \\
$\bar{\alpha} e^{i \varphi_{\alpha}}$ & $-\pi \rho u^{2} b(2 C(k)+i k+i k C(k))$ & $\pi \rho u^{2} b^{2}\left(C(k)-i k+i \frac{k}{2}+\frac{k^{2}}{8}\right)$ \\
$\bar{\kappa} e^{i \varphi_{\kappa}}$ & $-\pi \rho u^{2} b^{2}\left(C(k)+\frac{k^{2}}{8}\right)$ & $\frac{1}{2} \pi \rho u^{2} b^{3}\left(C(k)-1-i \frac{k}{2}\right)$ \\
$\bar{\kappa}_{2} e^{i \varphi_{\kappa 2}}$ & $-\frac{1}{4} \pi \rho u^{2} b^{3} C(k)$ & $\frac{1}{8} \pi \rho u^{2} b^{4}\left(C(k)-1-\frac{k^{2}}{24}\right)$ \\
$\bar{\kappa}_{3} e^{i \varphi_{\kappa 3}}$ & $-\frac{1}{24} \pi \rho u^{2} b^{4} C(k)$ & $\frac{1}{8} \pi \rho u^{2} b^{5}(C(k)-1)$ \\
\hline
\end{tabular}

It can be seen that the magnitude of the lift and pitching moment terms will grow to be very large as a function of reduced frequency. However, the phase angles of the lift and pitching moment relative to the airfoil motion change due to the value of $C(k)$. The phase also changes due to the quasi-steady parts as reduced frequency grows large. Theodorsen's function changes the lift and pitching moment phase as reduced frequency increases. At the point when reduced frequency reaches $k \approx 0.18$, the phase shift reaches a maximum and as reduced frequency continues to increase, the phase shift approaches it's value when reduced frequency is zero (see Figure 3.5). This phase shift effects only the circulatory lift. The phase of the noncirculatory lift changes as reduced frequency grows large due to the quasi-steady part of the lift and pitching moment. Figures 5.1 and 5.2 below show the phase of lift and pitching moment, respectively, for each of the first five airfoil shapes alone as a function of reduced frequency. In order to observe the entire range of reduced frequency, $k /(k+1)$ is used instead of $k$. Therefore when this value is zero, $k$ is zero, when it is $0.5, k$ is 1 , and when it is $1, k$ is infinity.

The plunge and pitch motion very easily show the effect Theodorsen's function has on the phase shift. The phase of the lift lags $90^{\circ}$ with the plunge motion initially and at low reduced frequencies the phase lags back even further as reduced frequency increases. For large 


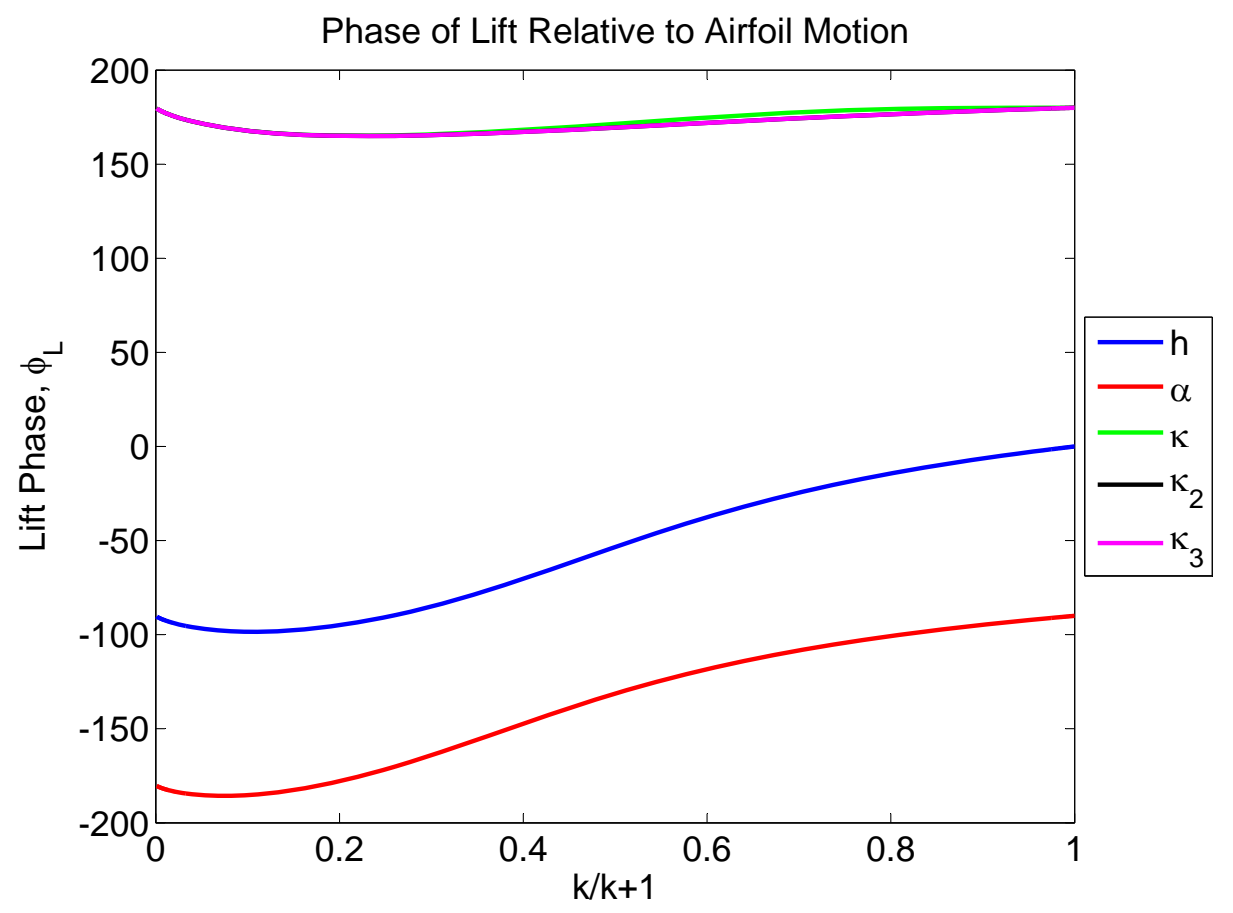

Figure 5.1: Phase of lift relative to airfoil motion

$k$, the terms with the highest order in $k$ in the lift expression become more prominent, while other terms become more insignificant. For the plunge case, the imaginary part of the lift expression becomes insignificant and the lift becomes in phase with the plunge motion. All the airfoil motion types considered show similar trends, which is due to the non-circulatory terms getting very large at high reduced frequency. This is because at high frequencies, the motion is dominated more by accelerations than circulation. The $\kappa_{2}$ term in Figure 5.1 directly coincides with the $\kappa_{3}$ curve and cannot be seen.

\subsubsection{Reduction to Steady Thin Airfoil Theory}

This theory is developed on the basis of small disturbances. Classical steady thin airfoil theory assumes the same. The lift and pitching moment results should easily reduce to steady thin airfoil theory when the frequency is zero. Therefore the lift terms in Table 5.1 reduce to the following lift and pitching moment expressions when the reduced frequency is zero. The value of $C(k)$ is 1 when $k=0$ and also $\varphi$ will be zero for each case. 


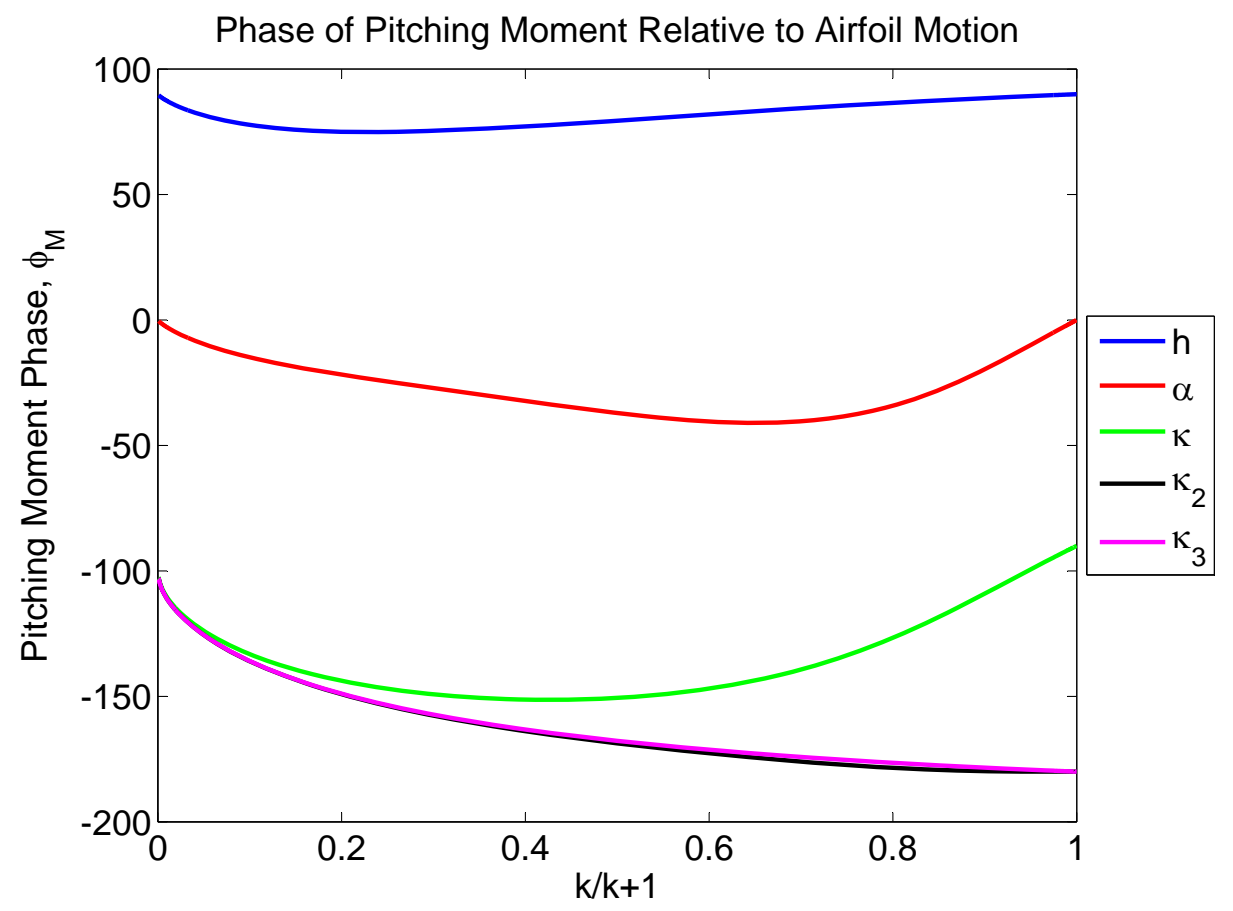

Figure 5.2: Phase of pitching moment relative to airfoil motion

$$
\begin{aligned}
L & =-2 \pi \rho v^{2} b \alpha-\pi \rho v^{2} b^{2} \kappa-\frac{1}{4} \pi \rho v^{2} b^{3} \kappa_{2}-\frac{1}{24} \pi \rho v^{2} b^{4} \kappa_{3} \\
M & =\pi \rho v^{2} b^{2} \alpha
\end{aligned}
$$

These equations are normalized by $\rho v^{2} b$ to give the lift and pitching moment coefficients,

$$
\begin{aligned}
C_{L} & =-2 \pi \alpha-\pi b \kappa-\frac{\pi}{4} b^{2} \kappa_{2}-\frac{\pi}{24} b^{3} \kappa_{3} \\
C_{M} & =\frac{\pi}{2} \alpha
\end{aligned}
$$

where, the pitching moment is about the midchord as stated in chapter 3 .

It is immediately obvious that the angle of attack term is identical to the thin airfoil solution. This is a useful result in getting a quick approximation for lift and pitching moment 
for any polynomial camberline shape desired. Due to the use of the Chebychev polynomials, the airfoil shape resulting in angle of attack results in a positive $\alpha$ being nose down. Therefore, since lift is positive up, when a negative $\alpha$ is used, which would be nose up, the lift is positive. The pitching moment is positive nose down. Therefore when a negative $\alpha$ is used, which is nose up, the pitching moment is negative, as is should be from thin airfoil theory. The plunge location, $h$, is positive up and $\kappa$ is concave up.

\section{$5.2 \quad$ Thrust Results}

The thrust, like the lift and pitching moment is also an oscillatory term. However, the value oscillates about an average value, and therefore the average thrust can be calculated for each type of motion, and combinations of each type of motion. The thrust is a quadratic function of the various motion types. This leads to cross coupling terms, as seen in Tables 3.5 through 3.8 .

The average thrust can be obtained by integrating the thrust over one time period, since the motion is harmonic. Harmonic motion takes the form $\sin (\omega t+\varphi)$. When employing the complex form of the airfoil motion, Eq. (3.38), the motion is specified as $\cos (\omega t+\varphi)+$

$i \sin (\omega t+\varphi)$. Only the sin or cos term is needed to specify the harmonic motion. Therefore either the real or imaginary part of the lift and pitching moment equations can be used to calculate the average thrust. Referring back to the equation for the thrust force, Eq. (3.46), the imaginary part of each component is used to derive the average expressions below. Keep in mind the real component would give the same result.

$$
T_{\text {average }}=\pi \rho b \frac{\omega}{2 \pi} \int_{0}^{\frac{2 \pi}{\omega}} \operatorname{Im}[S]^{2} d t+\frac{b \omega}{2 \pi} \int_{0}^{\frac{2 \pi}{\omega}} \int_{-1}^{1} \operatorname{Im}[\Delta p] \operatorname{Im}\left[\frac{\partial y}{\partial x}\right] d x d t
$$

The final expression for the average thrust is lengthy and therefore not displayed. However, the expressions for the individual airfoil motion types are much shorter due to the 
absence of coupled terms. The thrust due to the first five airfoil motion types individually are given as,

$$
\begin{aligned}
T_{h} & =\bar{h}^{2} \frac{k^{2} \pi \rho u^{2}}{b}\left(F^{2}+G^{2}\right) \\
T_{\alpha} & =\bar{\alpha}^{2} \frac{\pi \rho u^{2} b}{4}\left(\left(k^{2}+4\right) F^{2}-2\left(k^{2}+2\right) F+k^{2}-2 G k+G^{2}\left(k^{2}+4\right)\right) \\
T_{\kappa} & =\bar{\kappa}^{2} \frac{\pi \rho u^{2} b^{3}}{4}\left(F^{2}-F+G^{2}\right) \\
T_{\kappa_{2}} & =\bar{\kappa}_{2}^{2} \frac{\pi \rho u^{2} b^{5}}{64}\left(F^{2}-F+G^{2}\right) \\
T_{\kappa_{2}} & =\bar{\kappa}_{3}^{2} \frac{\pi \rho u^{2} b^{7}}{2304}\left(F^{2}-F+G^{2}\right)
\end{aligned}
$$

where, $C(k)=F+i G$.

The values of $F$ and $G$ are a complex combination of Bessel function which vary based on frequency. The derivation of these is given in Appendix A. For the plunge only case, the thrust is always positive[2]. The four airfoil shapes following may be positive or negative based on the reduced frequency. The thrust due to $\kappa, \kappa_{2}$ and $\kappa_{3}$ are indirectly functions of reduced frequency through $F$ and $G$. Therefore, these thrust values will approach a value rather than go to infinity as $k$ grows large. Figure 5.3 shows the thrust force coefficient for unit non-dimensional displacement/deformation due to each of the first five shapes individually. The thrust is a function of the square of the reduced frequency, and therefore the thrust coefficient is divided by $k^{2}$. The $x$-axis is $k /(k+1)$ so that values of reduced frequency between 0 and infinity can easily be seen. The amplitude of oscillation, $\bar{h}, \bar{\alpha} \ldots$ are set equal to 1.

Figure 5.3 shows that the plunge shape is the only motion with significant thrust, as shown by Garrick[2]. However, this shape along with the other shapes have cross coupling terms which can lead to added thrust depending on the phase of the motion relative to the plunge motion. The following four figures show combinations of plunge and the latter airfoil shapes at different phases to show how the thrust varies. The magnitude of the additional 


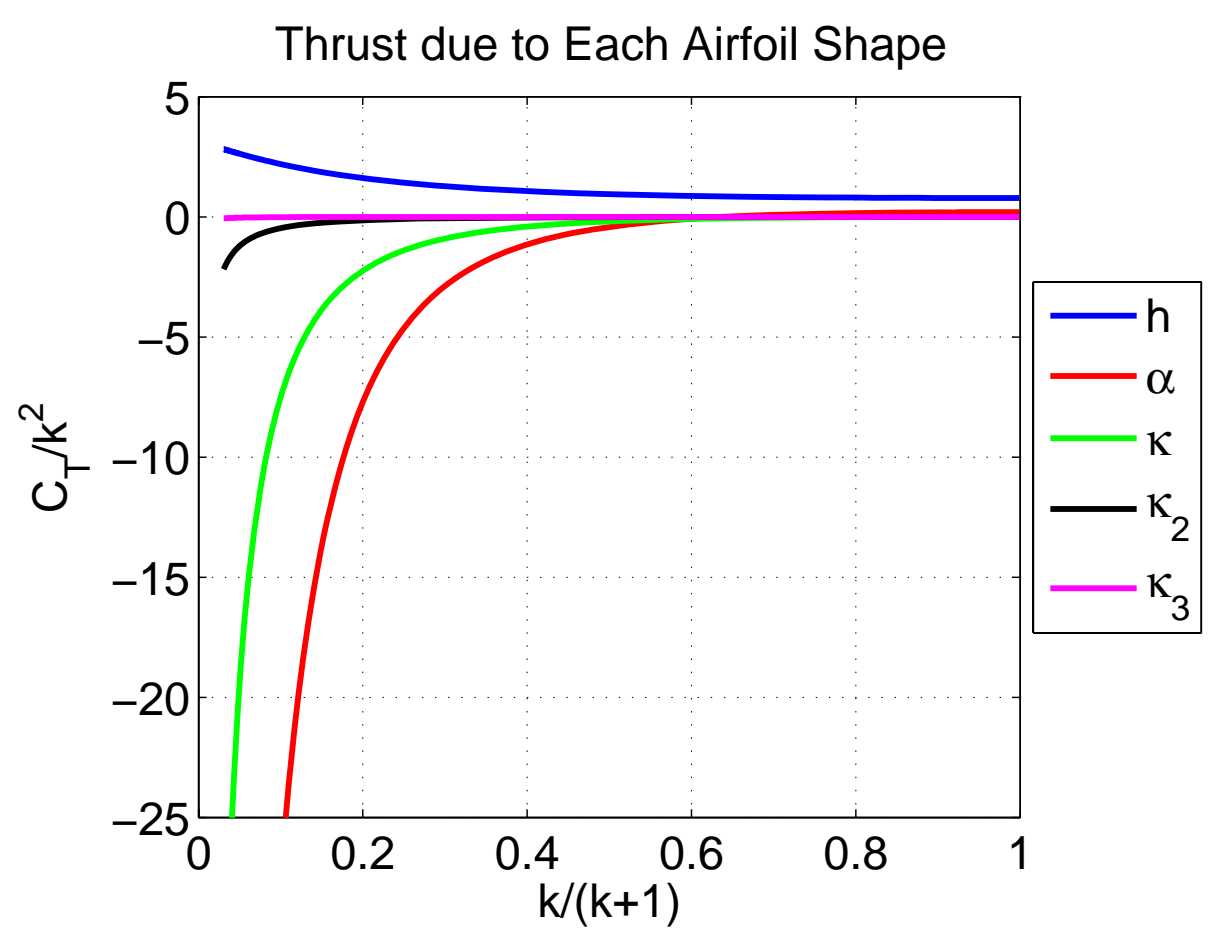

Figure 5.3: Thrust coefficient due to the first 5 airfoil shapes

deformation shape is $1 / 10$ the magnitude of the plunge shape for all figures.

It is obvious from Figure 5.4 that the addition of the $\alpha$ motion changes the thrust. The phase lead of $\pi / 2$ had the largest contribution to positive thrust. This phase lead is very similar to the way birds move their wings to generate propulsion[14]. Obviously this is not the only contribution, because birds also change the wing shape. Figure 5.5 shows a similar effect with the first camber shape, the $\pi / 2$ phase lead of the $\kappa$ motion relative to plunge is the best contribution to positive thrust. All the phases relative to plunge eventually converge to a positive thrust value at higher reduced frequencies. The same effect is seen in Figures 5.6 and 5.7 .

There are only certain phases where thrust is produced instead of drag. However, many of the cases which are drag will become thrust at higher reduced frequencies. This is simply due to the terms which are functions of accelerations, meaning they have a $k^{2}$ term. When this term is positive and when the reduced frequency becomes large enough, the drag becomes a thrust. Therefore all combinations of airfoil shapes with plunge eventually result 
Thrust Coefficient Due to $\mathrm{h}$ and $\alpha$

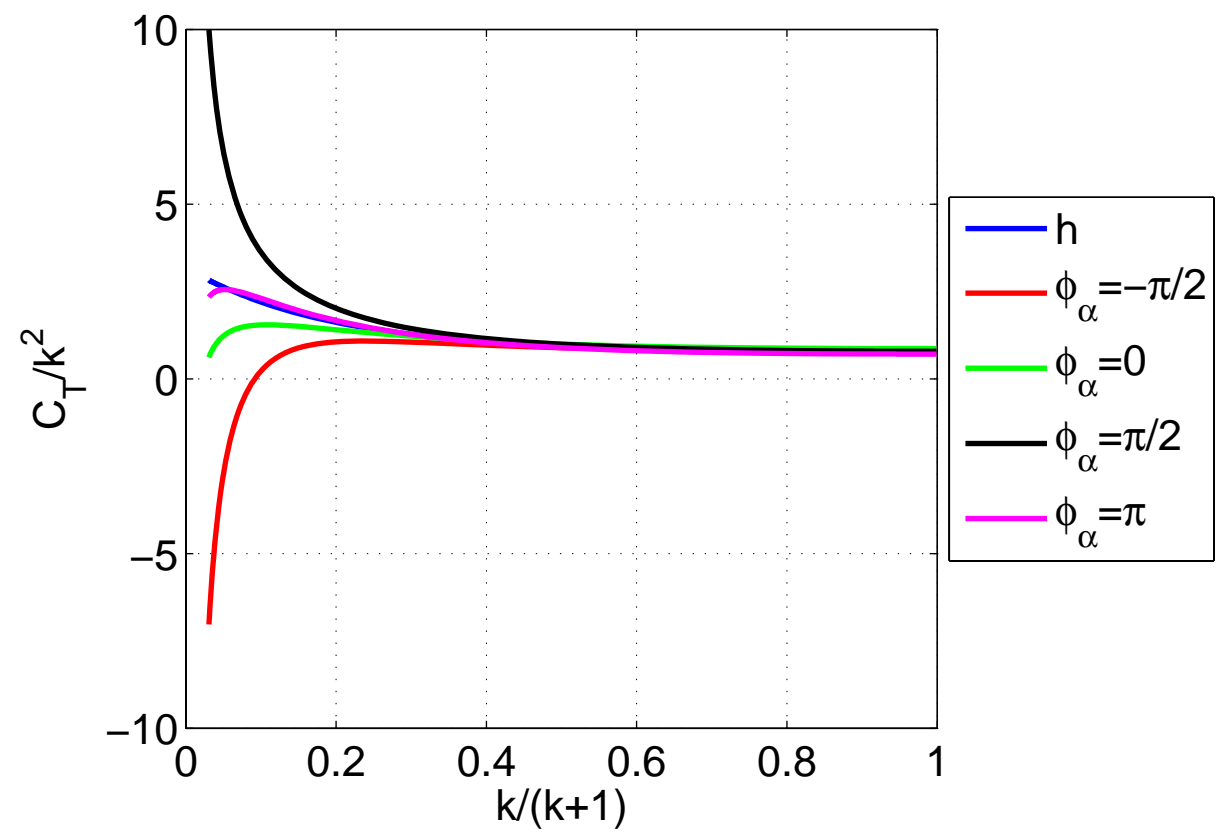

Figure 5.4: Thrust coefficient due to $h$ and $\alpha$

Thrust Coefficient Due to $\mathrm{h}$ and $\mathrm{\kappa}$

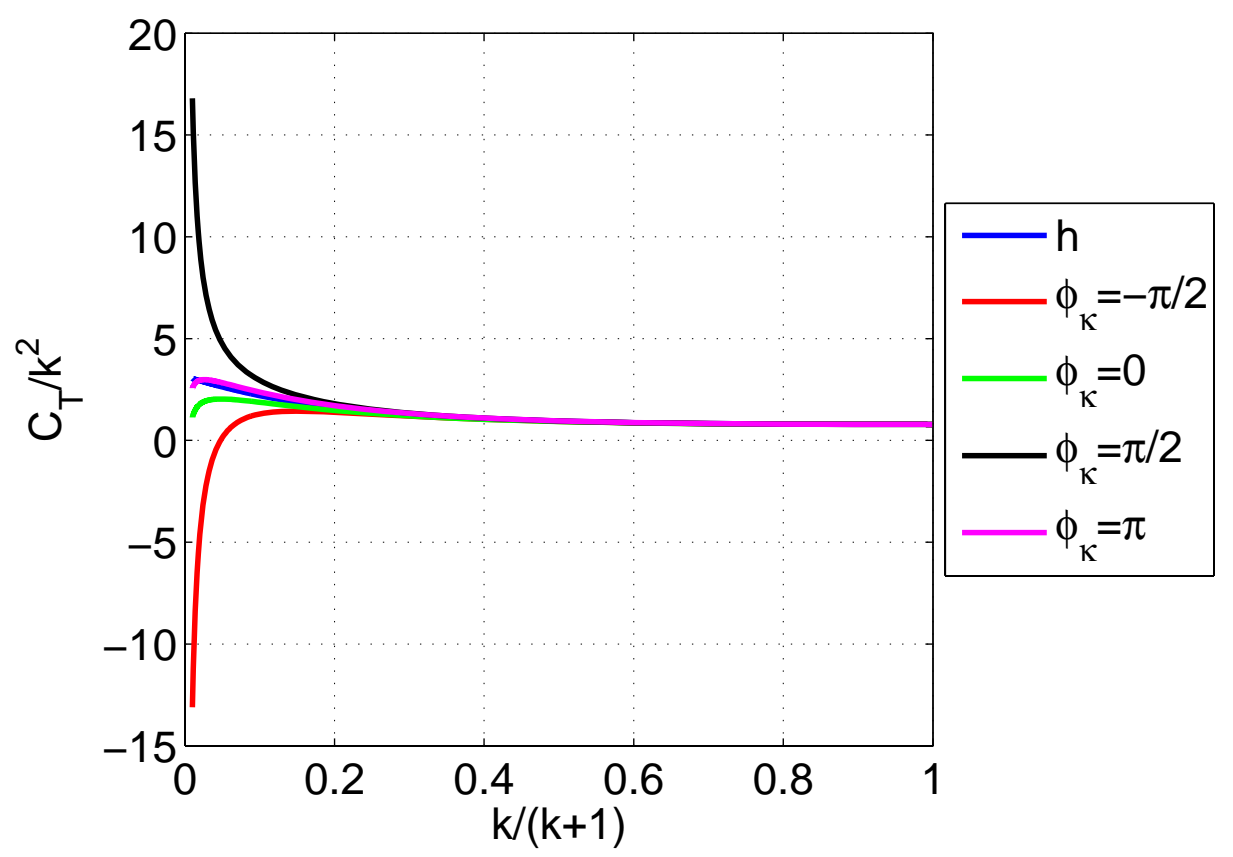

Figure 5.5: Thrust coefficient due to $h$ and $\kappa$ 


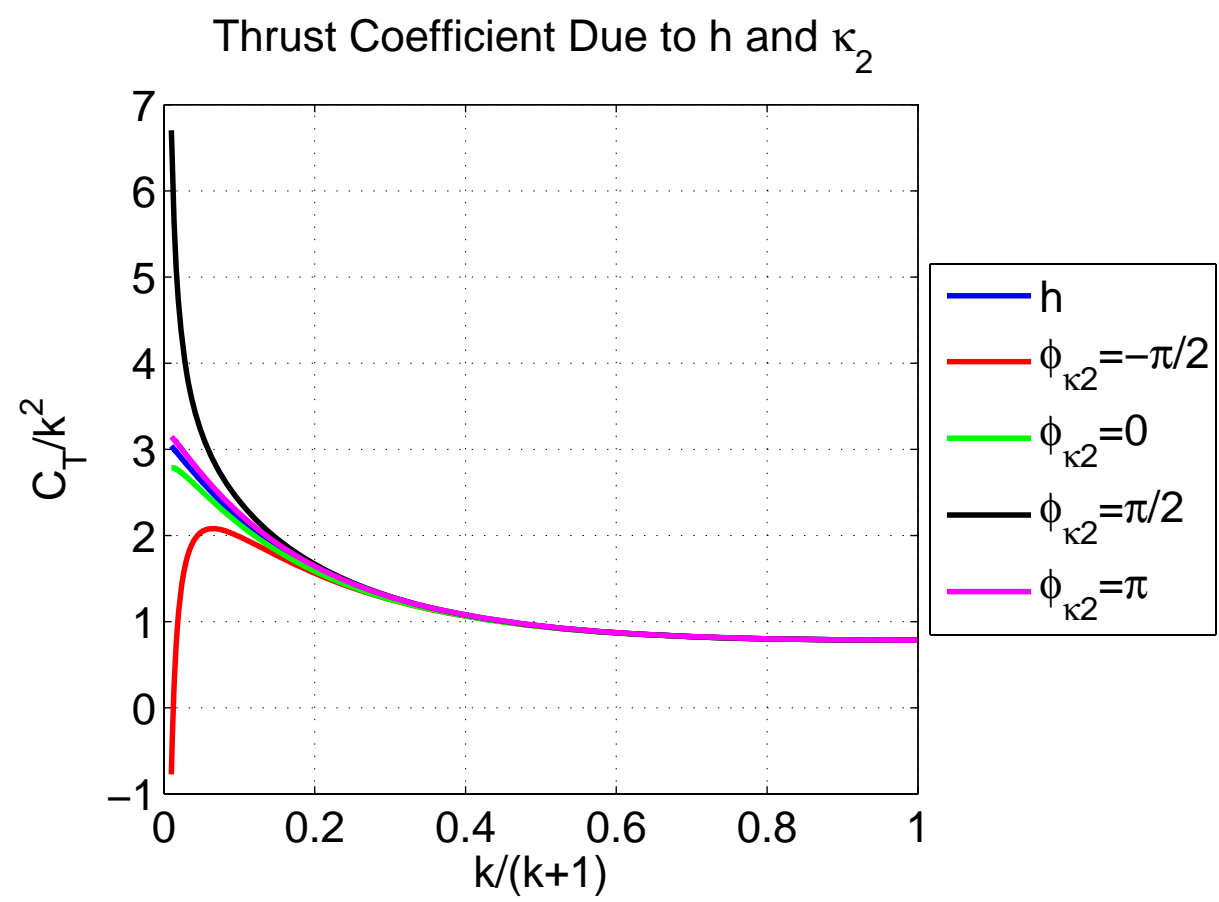

Figure 5.6: Thrust coefficient due to $h$ and $\kappa_{2}$

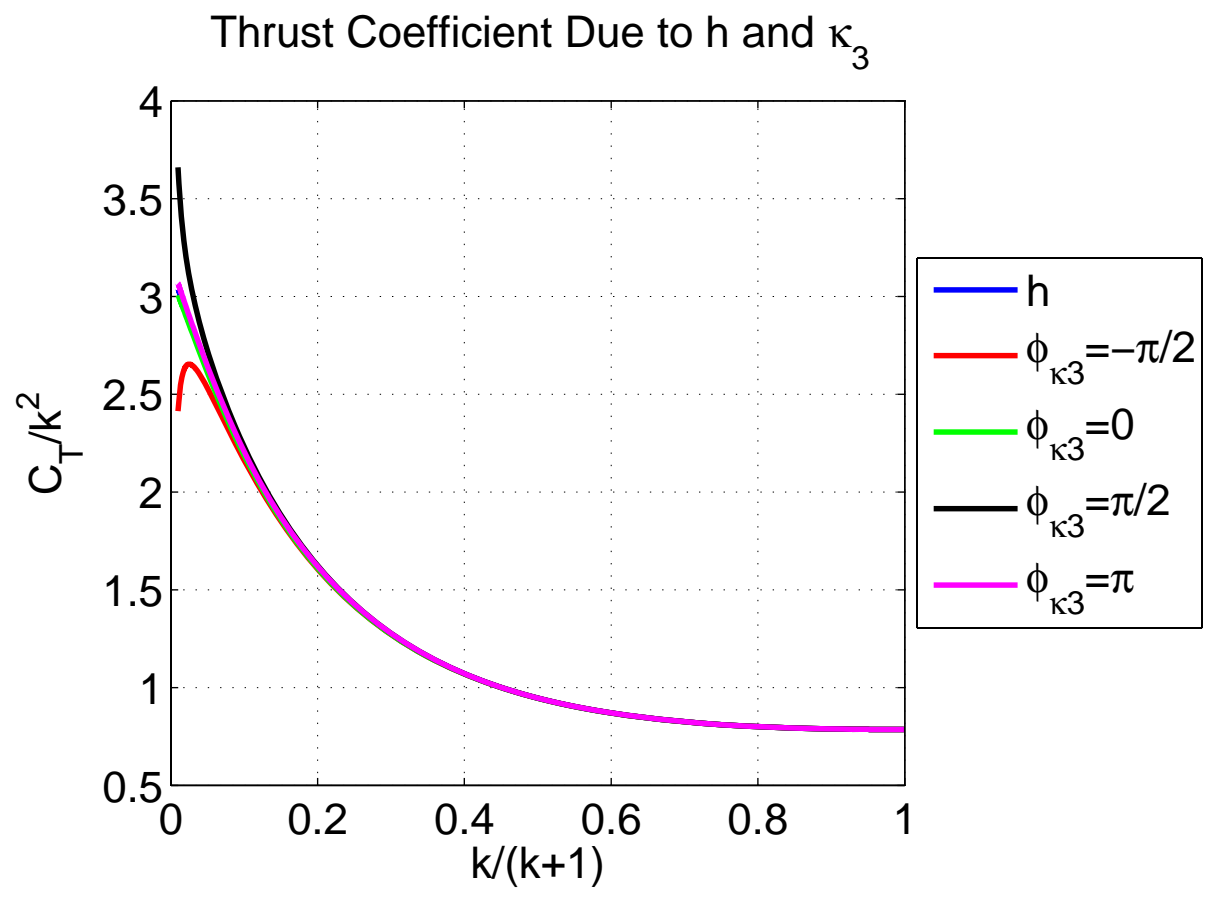

Figure 5.7: Thrust coefficient due to $h$ and $\kappa_{3}$ 
in thrust.

Combinations of airfoil shapes without plunge included are given in Figures 5.8 through 5.13. These airfoil shape combinations result in drag at low frequencies for every case, and for the shape combinations without $\alpha$, result in drag at all frequencies. However, as with the flows including plunge, the combinations with $\alpha$ becomes positive after some reduced frequency.

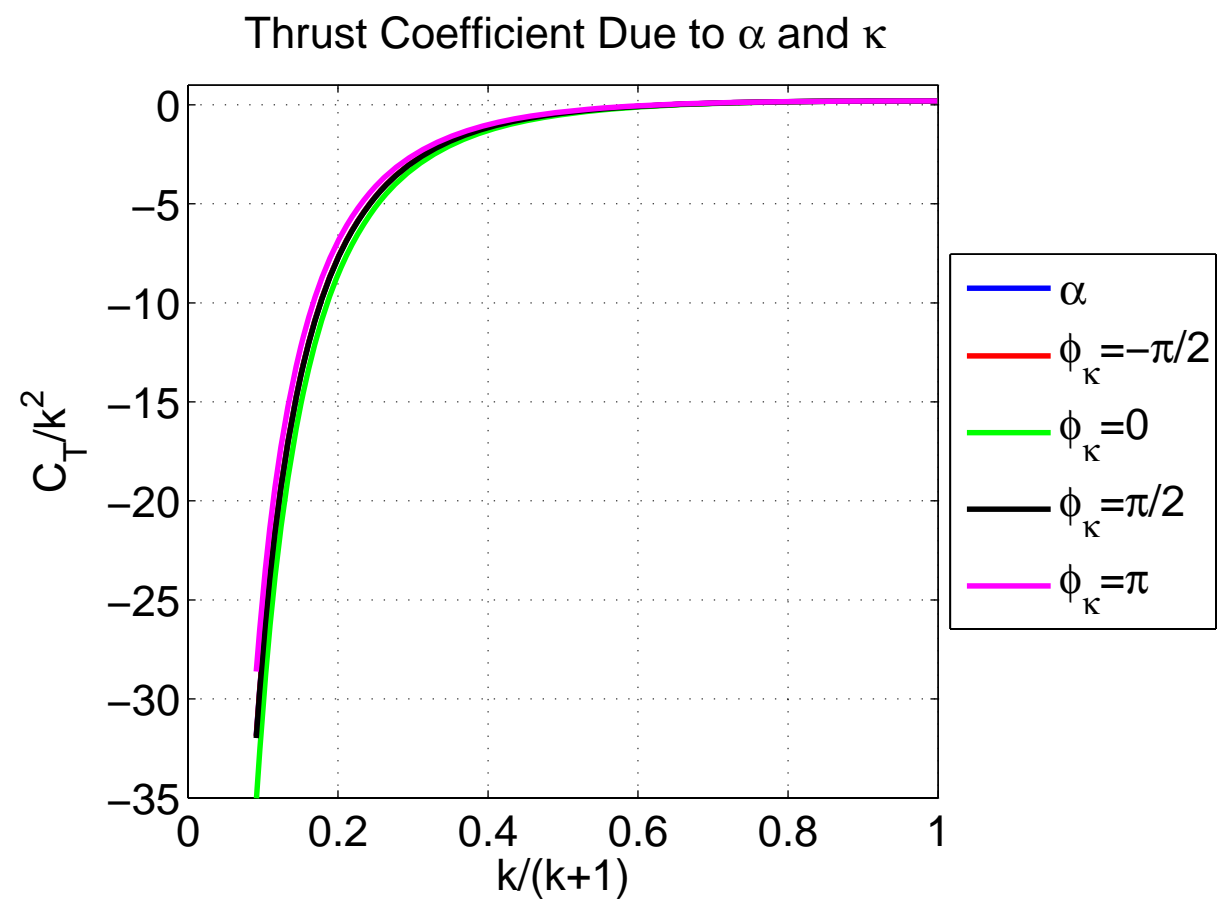

Figure 5.8: Thrust coefficient due to $\alpha$ and $\kappa$

Figures 5.8 through 5.13 show that every combination of the first five airfoil shapes without plunge will result in a drag rather than thrust at low reduced frequency. However, take for instance the flow consisting of $h, \kappa_{2}$, and $\kappa_{3}$. The combinations of $h$ and $\kappa_{2}$, and $h$ and $\kappa_{3}$ are both large, but the combination of $\kappa_{2}$ and $\kappa_{3}$ is a drag which is quite small. Therefore the combination of $h, \kappa_{2}$ and $\kappa_{3}$ may result in more thrust than $h$ and $\kappa_{2}$, and $h$ and $\kappa_{3}$ alone. There is much to be done in analyzing this; these results only take into account the magnitude of $1 / 10$ the plunge magnitude. Figures 5.14 through 5.17 show the combination of $h, \kappa_{2}$ and $\kappa_{3}$. These plots each have a constant $\kappa_{2}$ phase relative to plunge 
Thrust Coefficient Due to $\alpha$ and $\kappa_{2}$

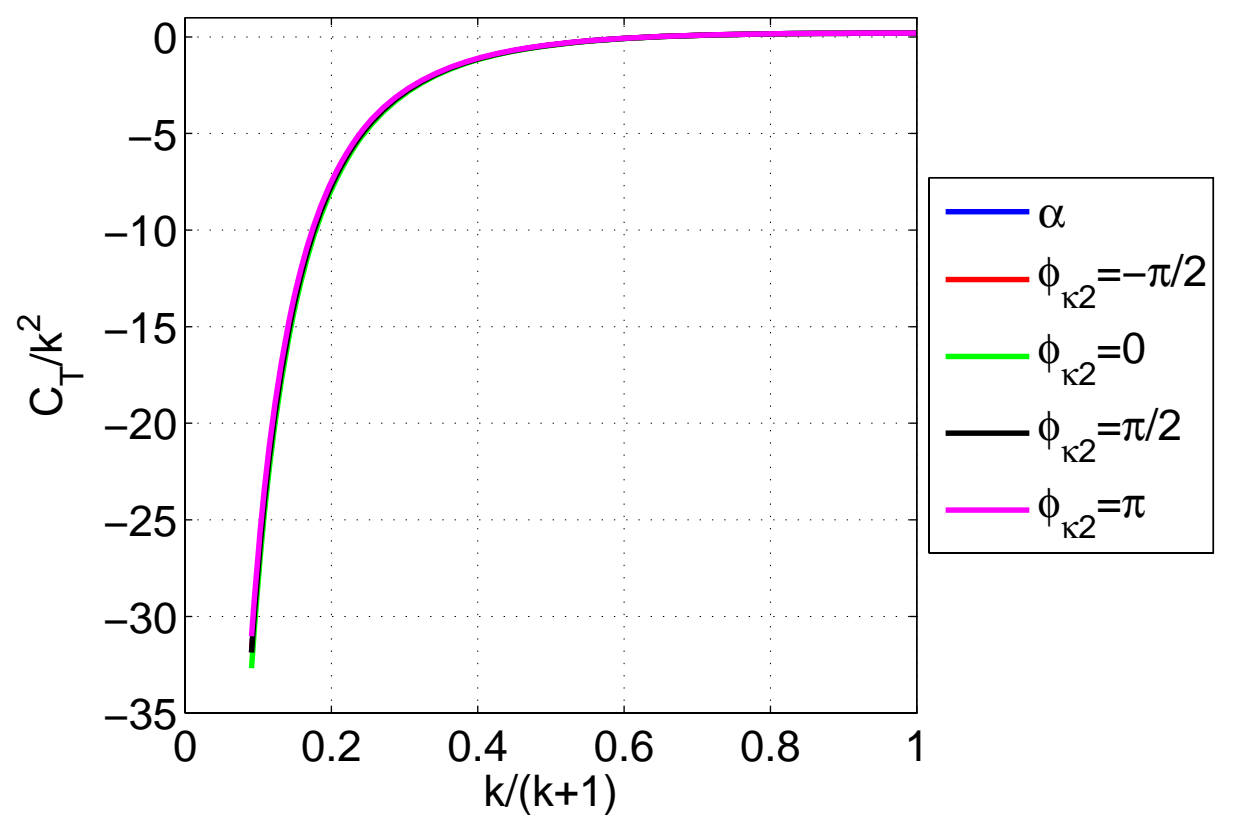

Figure 5.9: Thrust coefficient due to $\alpha$ and $\kappa_{2}$

and varying $\kappa_{3}$ phase.

Nearly every phase combination for the three deformations of Figures 5.14 through 5.17 results in thrust. There are several cases which did not result in thrust at very low frequencies. Take, for instance Figure 5.14; every curve results in drag rather than thrust at low frequencies, but becomes thrust at higher frequencies. Just like the curves for only one more shape and plunge, the effect of the positive $k^{2}$ takes over after a certain value of $k$ and the thrust force becomes positive. The value of reduced frequency this occurs at is dependent on the phase angle. It makes sense that there is no curve for this case which is always thrust because the phase of $\kappa_{2}$ is $-\pi / 2$. Figure 5.6 shows this phase angle to produce drag at low frequencies instead of thrust.

As seen with the combinations of $h$ and every other shape, the phase of the second motion relative to $h$ of $\pi / 2$ always produces thrust, and it follows that the combinations of $h, \kappa_{2}$, and $\kappa_{3}$ with this phase should be similar. Figure 5.16 shows this; every curve always produces thrust when $\phi_{\kappa 2}=\pi / 2$. 
Thrust Coefficient Due to $\alpha$ and $\kappa_{3}$

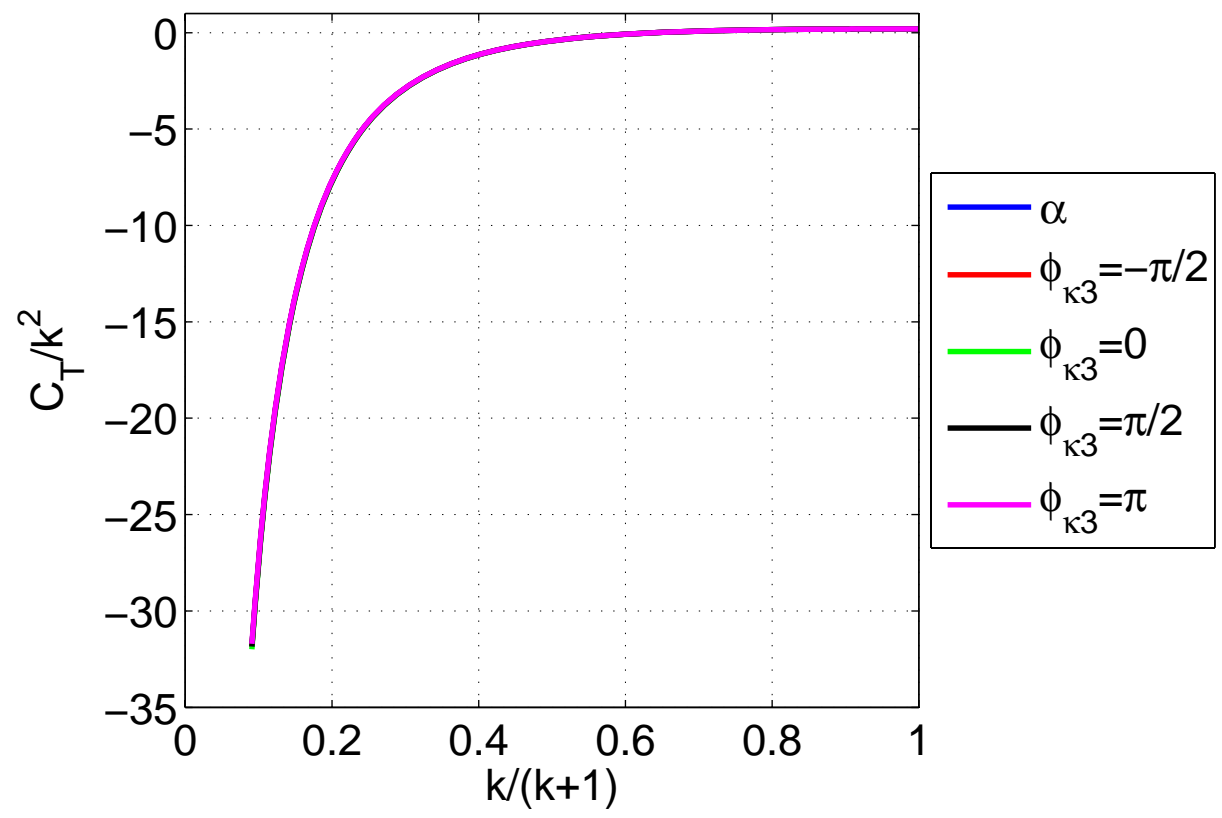

Figure 5.10: Thrust coefficient due to $\alpha$ and $\kappa_{3}$

Thrust Coefficient Due to $\kappa$ and $\kappa_{2}$

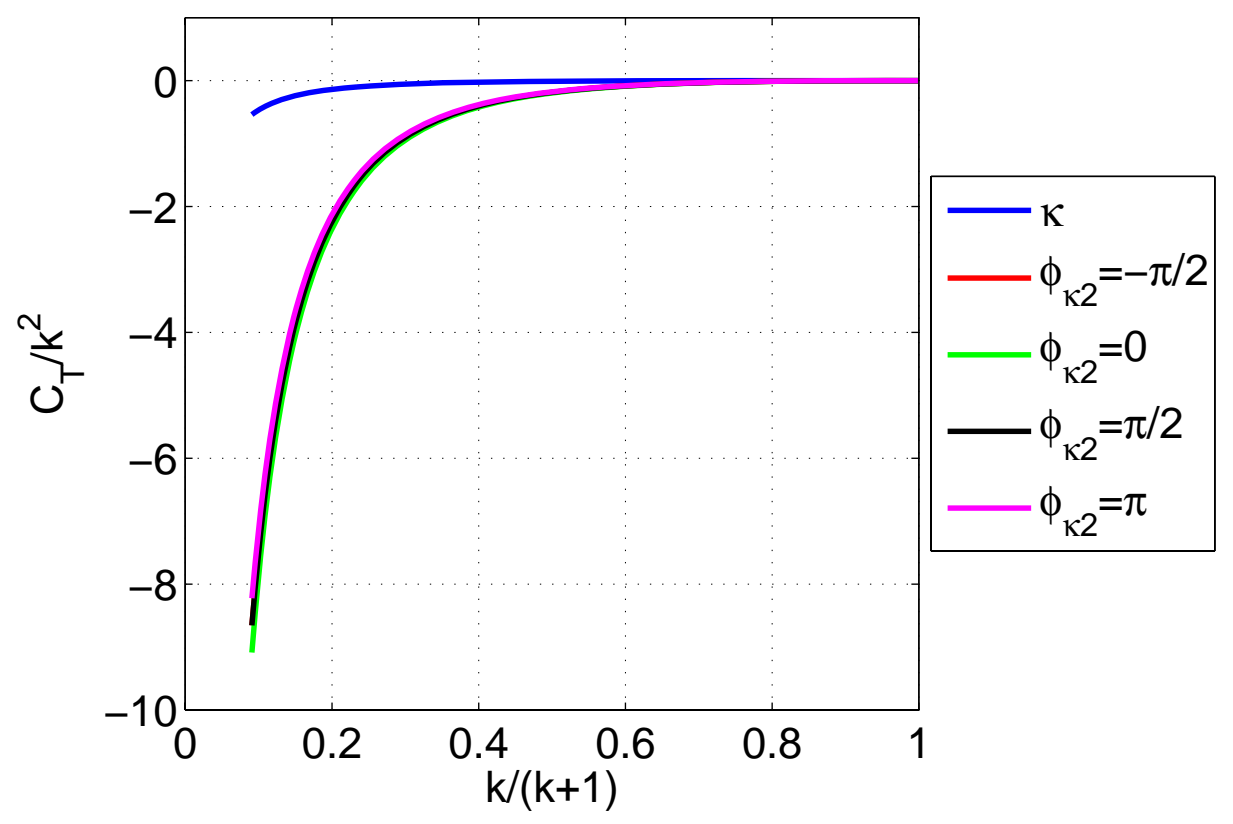

Figure 5.11: Thrust coefficient due to $\kappa$ and $\kappa_{2}$ 
Thrust Coefficient Due to $\kappa$ and $\kappa_{2}$

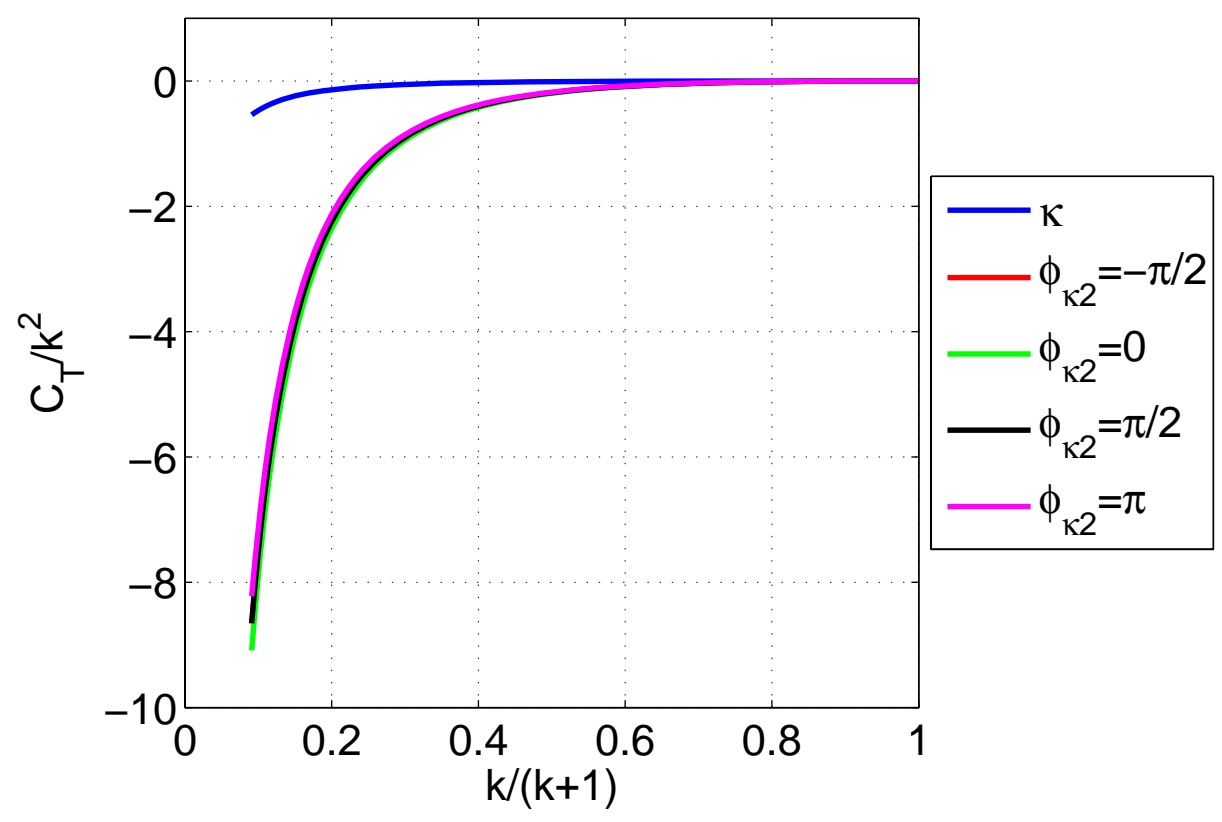

Figure 5.12: Thrust coefficient due to $\kappa$ and $\kappa_{3}$

Thrust Coefficient Due to $\kappa_{2}$ and $\kappa_{3}$

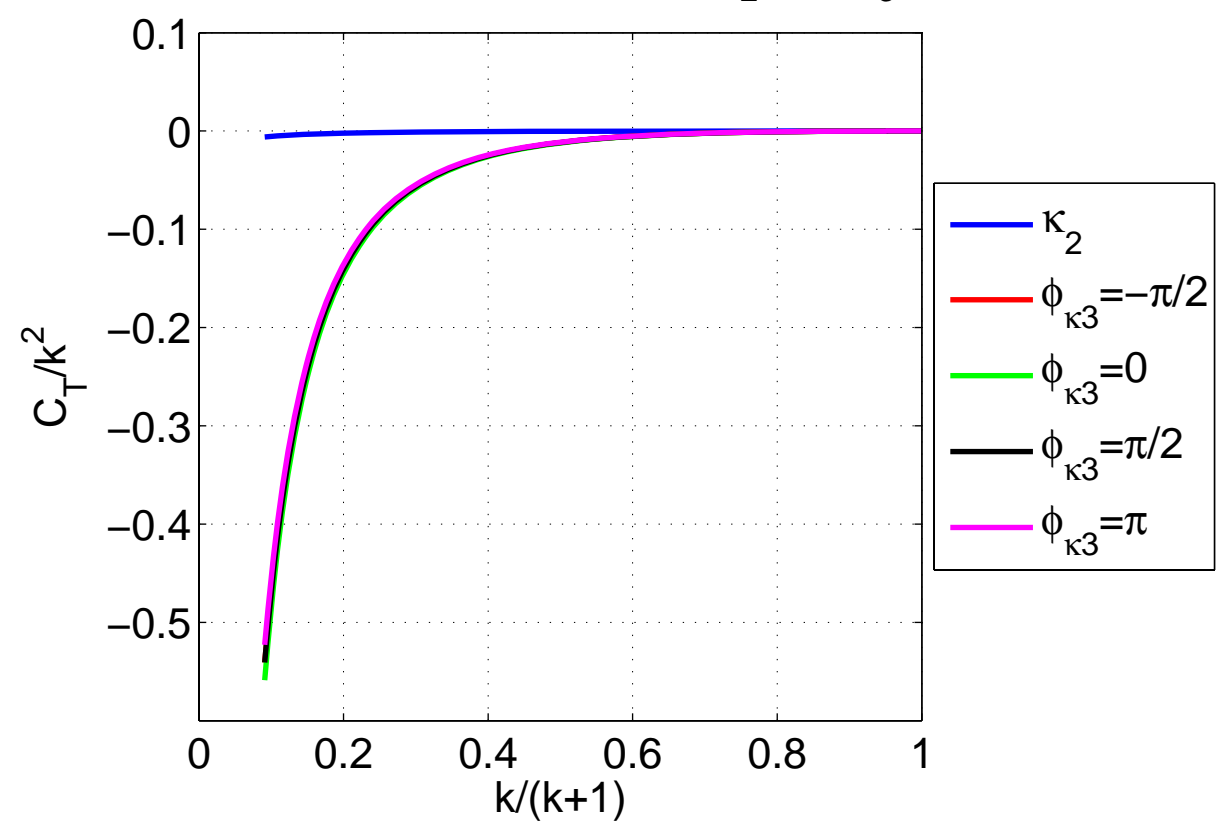

Figure 5.13: Thrust coefficient due to $\kappa_{2}$ and $\kappa_{3}$ 


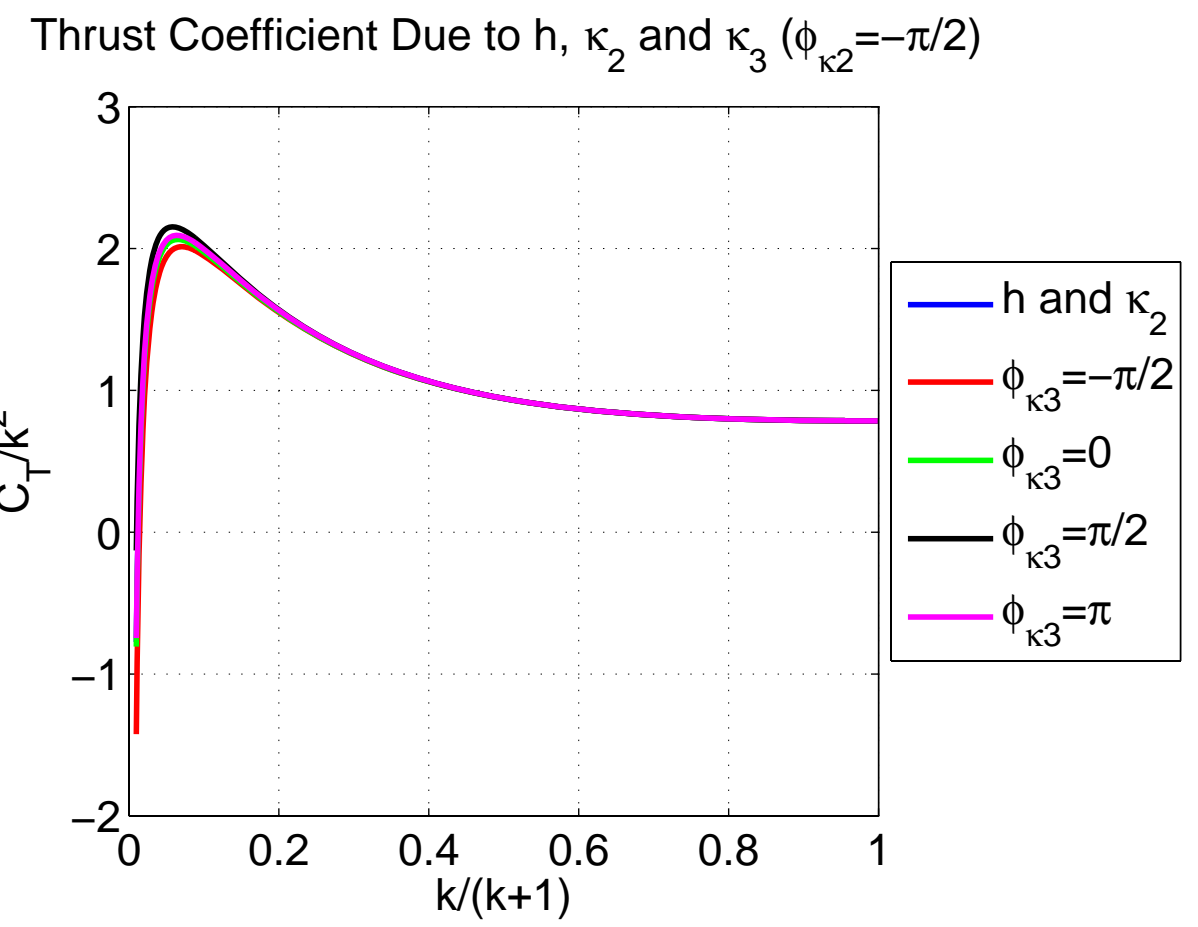

Figure 5.14: Thrust coefficient due to $h, \kappa_{2}$ and $\kappa_{3}\left(\phi_{\kappa 2}=-\pi / 2\right)$

Thrust Coefficient Due to $h, \kappa_{2}$ and $\kappa_{3}\left(\phi_{\mathrm{K} 2}=0\right)$

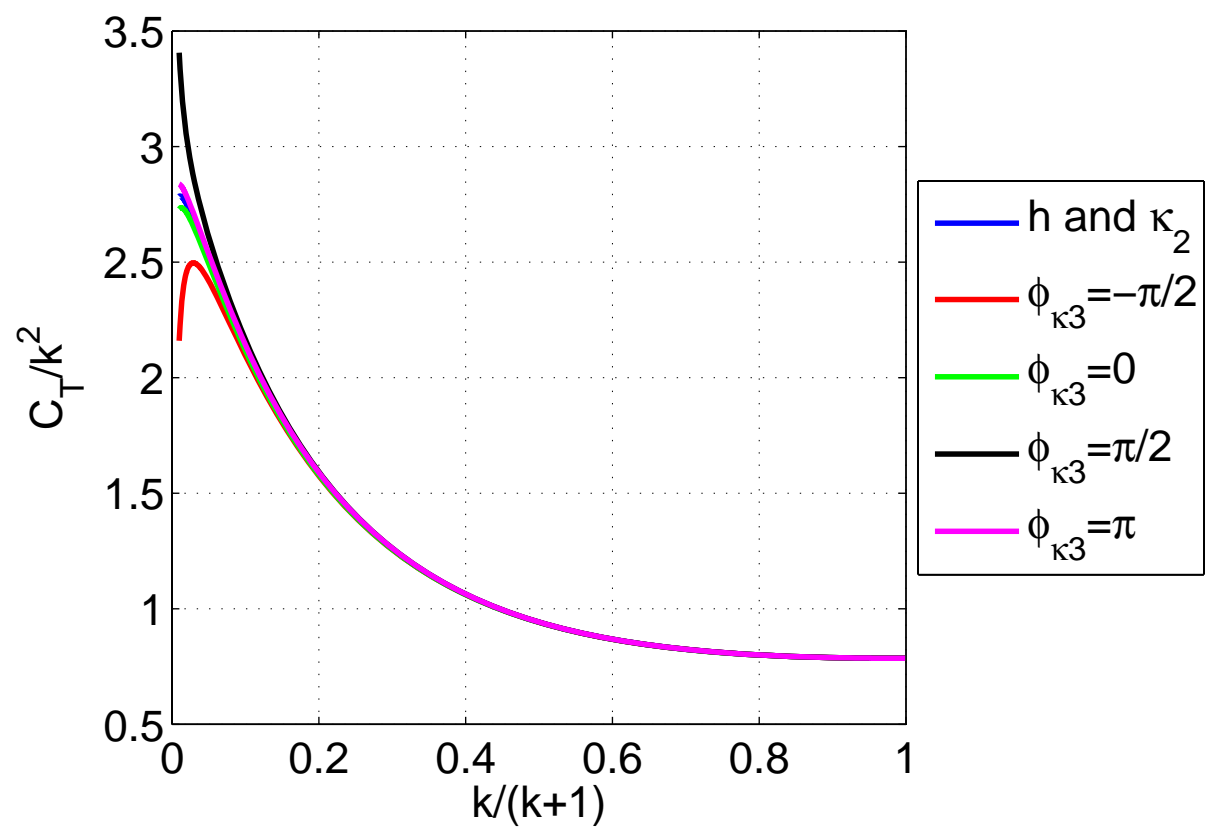

Figure 5.15: Thrust coefficient due to $h, \kappa_{2}$ and $\kappa_{3}\left(\phi_{\kappa 2}=0\right)$ 
Thrust Coefficient Due to $\mathrm{h}, \kappa_{2}$ and $\kappa_{3}\left(\phi_{\kappa 2}=\pi / 2\right)$

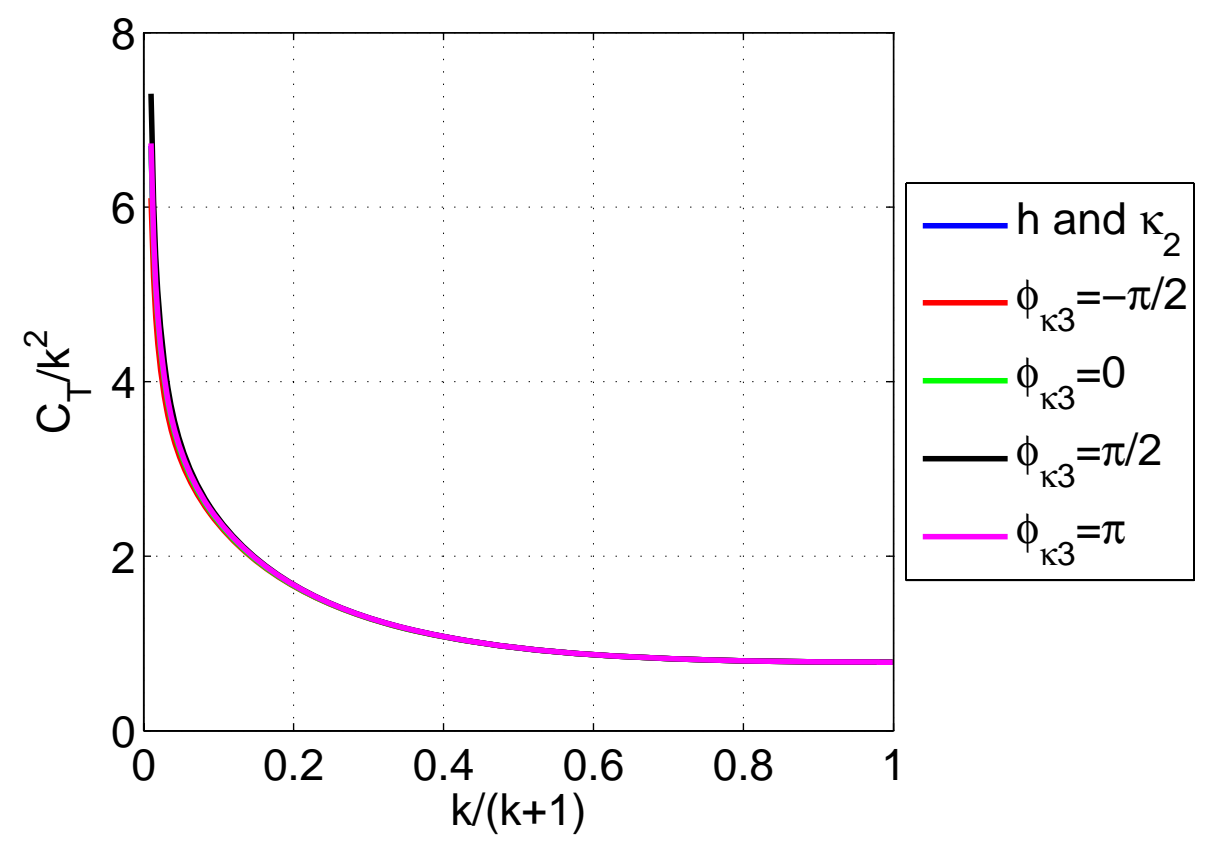

Figure 5.16: Thrust coefficient due to $h, \kappa_{2}$ and $\kappa_{3}\left(\phi_{\kappa 2}=\pi / 2\right)$

Thrust Coefficient Due to $\mathrm{h}, \kappa_{2}$ and $\kappa_{3}\left(\phi_{\mathrm{k} 2}=\pi\right)$

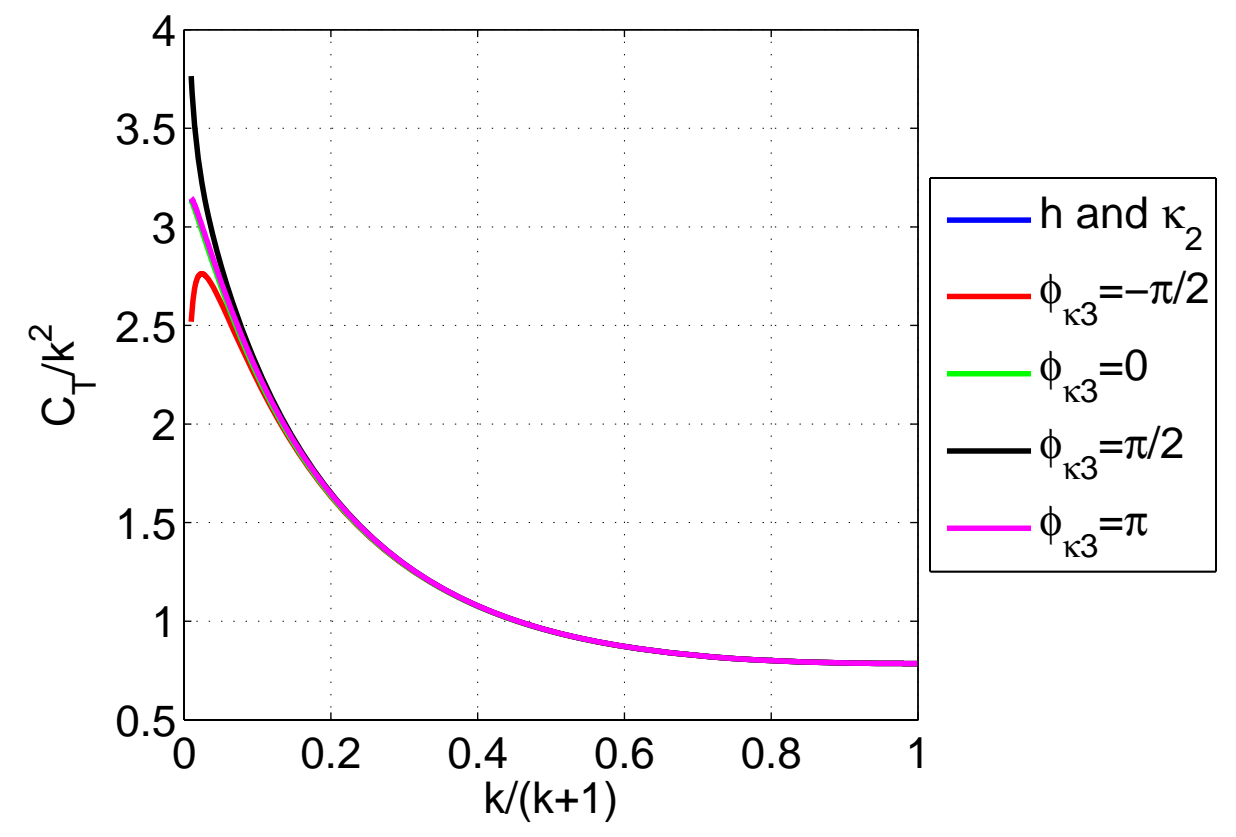

Figure 5.17: Thrust coefficient due to $h, \kappa_{2}$ and $\kappa_{3}\left(\phi_{\kappa 2}=\pi\right)$ 


\section{Chapter 6}

\section{Conclusions and Future Work}

\subsection{Conclusions}

The unsteady aerodynamics of deformable airfoils was addressed in this thesis. The deformation of the airfoil is represented by a series of Chebychev polynomials. The aerodynamics model was developed using potential flow aerodynamics. The model consisted of a sheet of sources/sinks stretched along a circle. The Joukowski conformal mapping technique was used to map the flow around the circle to the flow around a flat plate. The strength of the sheet was determined by the no penetration boundary condition. The circulation was modeled as a sheet of vortices extending to infinity in the wake, and a sheet on the body for the bound circulation. The strength of the sheet was determined by the Kutta condition. These boundary conditions were used to derive the lift, pitching moment and thrust on a flat plate which can pitch, plunge, and deform in any shape. The key conclusions based on a case study investigating the resulting equations are as follows.

- Theodorsen's function reduces the magnitude of the lift due to circulation as reduced frequency increases.

- Theodorsen's function shifts the phase of the circulatory lift with respect to the motion for all airfoil deformation. The magnitude of the phase shift is dependent on the reduced 
frequency.

- As shown by Garrick, plunging of a flat plate always leads to thrust.

- Combinations of plunging with other displacement/deformation shapes leads to changes in the thrust as compared to pure plunging. When the deformation leads the plunge by $\pi / 2$ there is an increase in the magnitude of the thrust as compared to plunging only.

- The magnitude of the pitching and deformation motion relative to the plunge magnitude affects the thrust.

\subsection{Future Work}

The method derived in this thesis yields the lift force, pitching moment, and thrust for an airfoil deforming harmonically. The derived theory is an inviscid, two dimensional theory. Many flapping wing MAV's will have motion outside the regime of small disturbances, and likely have significant three dimensional effects. However, steady aerodynamics has shown the use of thin airfoil theory to be quite helpful in conceptual design of aircraft. Therefore it is expected that the theory developed in this thesis will be helpful in preliminary design of flapping wing aircraft. Further, the theory will be helpful in understanding how birds and insects generate lift and propulsion. The present theory can be used as the foundation to develop a more advanced theory. There are numerous possibilities for other work to be done in conjunction with this work and as extensions to this work. Several paths for future work include the following.

- Derive the lift, thrust and pitching moment for a different set of orthogonal functions.

- Investigate the effect of mean camber and thickness by changing the location of the Joukowski circle being mapped to a location other than the origin.

- Investigate the effect of dynamically moving the circle being mapped vertically. 
- Develop methodology for optimizing the deformation shape for thrust generation (magnitude and efficiency).

- Couple the unsteady aerodynamic model with membrane structural model to develop a methodology for aeroelastic analysis of MAV wings.

- Investigate the lift, thrust and pitching moment during transient motion. 


\section{References}

[1] Theodorsen, T., "General Theory of Aerodynamic Instability and the Mechanism of Flutter," NACA TR No. 496, May 1934.

[2] Garrick, I. E., "Propulsion of a Flapping and Oscillating Airfoil," NACA TR No. 567, May 1936.

[3] von Kármán, T. and Burgers, J. M., General Aerodynamic Theory-Perfect Fluids, Vol. II, Julius Springer, Berlin, 1935.

[4] Katz, J. and Plotkin, A., Low-Speed Aerodynamics, Cambridge University Press, 2002.

[5] Wagner, H., "Über die Entstehung des dynamischen Auftriebes von Tragflügeln," February 1925 , pp. 17-35.

[6] Garrick, I. E., "On Some Reciprical Relations in the Theory of Nonstationary Flows," NACA TR No. 629, March 1938.

[7] Küssner, H. G., "Zusammenfassender Bericht über den instationaren Auftrieb von Flügeln," December 1936, pp. 410-424.

[8] von Kármán and Sears, "Airfoil Theory for Non-Uniform Motion," Journal of the Aeronautical Sciences, Vol. 5, No. 10, 1938, pp. 379-390.

[9] Sears, W. R., "Operational Methods in the Theory of Airfoils in Non-Uniform Motion," Journal of the Franklin Institute, Vol. 230, July 1940, pp. 95-111. 
[10] Isaacs, R., "Airfoil Theory for Flows of Variable Velocity," Journal of the Aeronautical Sciences, Vol. 12, No. 1, 1945, pp. 113-117.

[11] Isaacs, R., "Airfoil Theory for Rotary Wing Aircraft," Journal of the Aeronautical Sciences, Vol. 13, No. 4, 1946, pp. 218-220.

[12] Loewy, R., "A Two-Dimensional Approximation to the Unsteady Aerodynamics of Rotary Wings," Journal of the Aeronautical Sciences, Vol. 24, No. 2, February 1957, pp. 8192.

[13] Greenberg, J. M., "Airfoil in Sinusoidal Motion in a Pulsating Stream," NACA TN No. 1326, June 1947.

[14] Zbikowski, R., "On aerodynamic modelling of an insect-like flapping wing in hover for micro air vehicles," Philosophical Transactions of the Royal Society London, Series A (Mathematical, Physical and Engineering Sciences), Vol. 360, No. 1791, 2002/02/15, pp. $273-90$.

[15] Azuma, A. and Okamoto, M., "Theoretical Study of the Two-dimensional Aerodynamic Charecteristics of Unsteady Wings," Journal of Theoretical Biology, November 2004.

[16] Patil, M. J., "From fluttering wings to flapping flight: The energy connection," Journal of Aircraft, Vol. 40, No. 2, 2003, pp. 270 - 276.

[17] Peters, D. A., Hsieh, M.-C. A., and Torrero, A., "A state-space airloads theory for flexible airfoils," Annual Forum Proceedings - AHS International, Vol. III, Phoenix, AZ, United states, 2006, pp. 1806 - 1823.

[18] Peters, D., "Two-dimensional incompressible unsteady airfoil theory-An overview," Journal of Fluids and Structures, Vol. 24, No. 3, 2008, pp. 295 - 312.

[19] Johnston, C., "Review, Extension, and Application of Unsteady Thin Airfoil Theory," CIMSS Report No. 04-101, Center for Intelligent Material Systems and Structures, Virginia Polytechnic Institute and State Univeristy, Blacksburg, VA, August 2004. 
[20] Glegg, S. and Devenport, W., "Unsteady loading on an airfoil of arbitrary thickness," Journal of Sound and Vibration, Vol. 319, No. 3-5, 2009, pp. 1252 - 70.

[21] Jones, R. T., "The Unsteady Lift of a Wing of Finite Aspect Ratio," NACA TR No. 681, June 1939.

[22] Hernandes, F. and Soviero, P. A. D. O., "A numerical model for thin airfoils in unsteady compressible arbitrary motion," Journal of the Brazilian Society of Mechanical Sciences and Engineering, Vol. 29, No. 3, 2007, pp. 253 - 261.

[23] Bisplinghoff, R., A. H. and Halfman, R., Aeroelasticity, Addison-Wesley, 1955. 


\section{Appendix A: Theodorsen's Function}

The complete derivation of Theodorsen's function is given in reference [1]. This section intends to highlight some of the aspects of the derivation and show the real and imaginary parts used in the calculation of the average thrust force in the case study of chapter 5 . Integral forms of Bessel functions $J_{0}, J_{1}, Y_{0}$, and $Y_{1}$ derived by Theodorsen are given below in Eq. (A.1).

$$
\begin{aligned}
-\frac{\pi}{2} Y_{0}(k) & =\int_{1}^{\infty} \frac{\cos k x_{0}}{\sqrt{x_{0}^{2}-1}} d x_{0} \\
\frac{\pi}{2} J_{0}(k) & =\int_{1}^{\infty} \frac{\sin k x_{0}}{\sqrt{x_{0}^{2}-1}} d x_{0} \\
-\frac{\pi}{2} Y_{1}(k) & =\int_{1}^{\infty} \frac{x_{0} \sin k x_{0}}{\sqrt{x_{0}^{2}-1}} d x_{0} \\
-\frac{\pi}{2} J_{1}(k) & =\int_{1}^{\infty} \frac{x_{0} \cos k x_{0}}{\sqrt{x_{0}^{2}-1}} d x_{0}
\end{aligned}
$$

Theodorsen's function is rewritten in Eq. (A.2) below.

$$
C(k)=\frac{\int_{1}^{\infty} \frac{x_{0}}{\sqrt{x_{0}^{2}-1}} e^{-i k x_{0}} d x_{0}}{\int_{1}^{\infty} \frac{x_{0}}{\sqrt{x_{0}^{2}-1}} e^{-i k x_{0}} d x_{0}+\int_{1}^{\infty} \frac{1}{\sqrt{x_{0}^{2}-1}} e^{-i k x_{0}} d x_{0}}
$$


Using Euler's equation, the exponential is written in the trigonometric form.

$$
C(k)=\frac{\int_{1}^{\infty} \frac{x_{0} \cos k x_{0}}{\sqrt{x_{0}^{2}-1}} d x_{0}-i \int_{1}^{\infty} \frac{\frac{x_{0} \sin k x_{0}}{\sqrt{x_{0}^{2}-1}} d x_{0}}{\int_{1}^{\infty} \frac{x_{0} \cos k x_{0}}{\sqrt{x_{0}^{2}-1}} d x_{0}-i \int_{1}^{\infty} \frac{x_{0} \sin k x_{0}}{\sqrt{x_{0}^{2}-1}} d x_{0}+\int_{1}^{\infty} \frac{\cos k x_{0}}{\sqrt{x_{0}^{2}-1}} d x_{0}-i \int_{1}^{\infty} \frac{\sin k x_{0}}{\sqrt{x_{0}^{2}-1}} d x_{0}}}{}
$$

Combining Eqs. (A.1) and (A.3) Theodorsen's function is written in terms of Bessel functions.

$$
\begin{aligned}
C(k) & =\frac{-\frac{\pi}{2} J_{1}(k)+i \frac{\pi}{2} Y_{1}(k)}{-\frac{\pi}{2} J_{1}(k)+i \frac{\pi}{2} Y_{1}(k)-\frac{\pi}{2} Y_{0}(k)-i \frac{\pi}{2} J_{0}(k)} \\
& =\frac{-J_{1}(k)+i Y_{1}(k)}{-\left(J_{1}(k)+Y_{0}(k)\right)+i\left(Y_{1}(k)-J_{0}(k)\right)}
\end{aligned}
$$

Eq. (A.4) is split up into real and imaginary parts in Eq. (A.5).

$$
\begin{aligned}
C(k) & =F(k)+i G(k) \\
F(k) & =\frac{J_{1}(k)\left(J_{1}(k)+Y_{0}(k)\right)+Y_{1}(k)\left(Y_{1}(k)-J_{0}(k)\right)}{\left(J_{1}(k)+Y_{0}(k)\right)^{2}+\left(Y_{1}(k)-J_{0}(k)\right)^{2}} \\
G(k) & =-\frac{Y_{1}(k) Y_{0}(k)+J_{1}(k) J_{0}(k)}{\left(J_{1}(k)+Y_{0}(k)\right)^{2}+\left(Y_{1}(k)-J_{0}(k)\right)^{2}}
\end{aligned}
$$

This form of Theodorsen's function much more easily shows it's effect on reducing the circulatory lift magnitude and shifting the phase of the circulatory lift relative to the motion. Recalling Eq. (3.42), the relationship for Theodorsen's function can be written in terms of Hankel functions. The numerator and first two terms of the denominator of Eq. (A.4) become $-H_{1}^{(2)}(k)$. The last two terms of the denominator are $-i H_{0}^{(2)}(k)$. Therefore the alternate for of Theodorsen's function is written as Eq. (A.6). 


$$
C(k)=\frac{H_{1}^{(2)}(k)}{H_{1}^{(2)}(k)+i H_{0}^{(2)}(k)}
$$

Figure A.1 below shows a plot of the real and imaginary parts of Theodorsen's function as a function of reduced frequency.

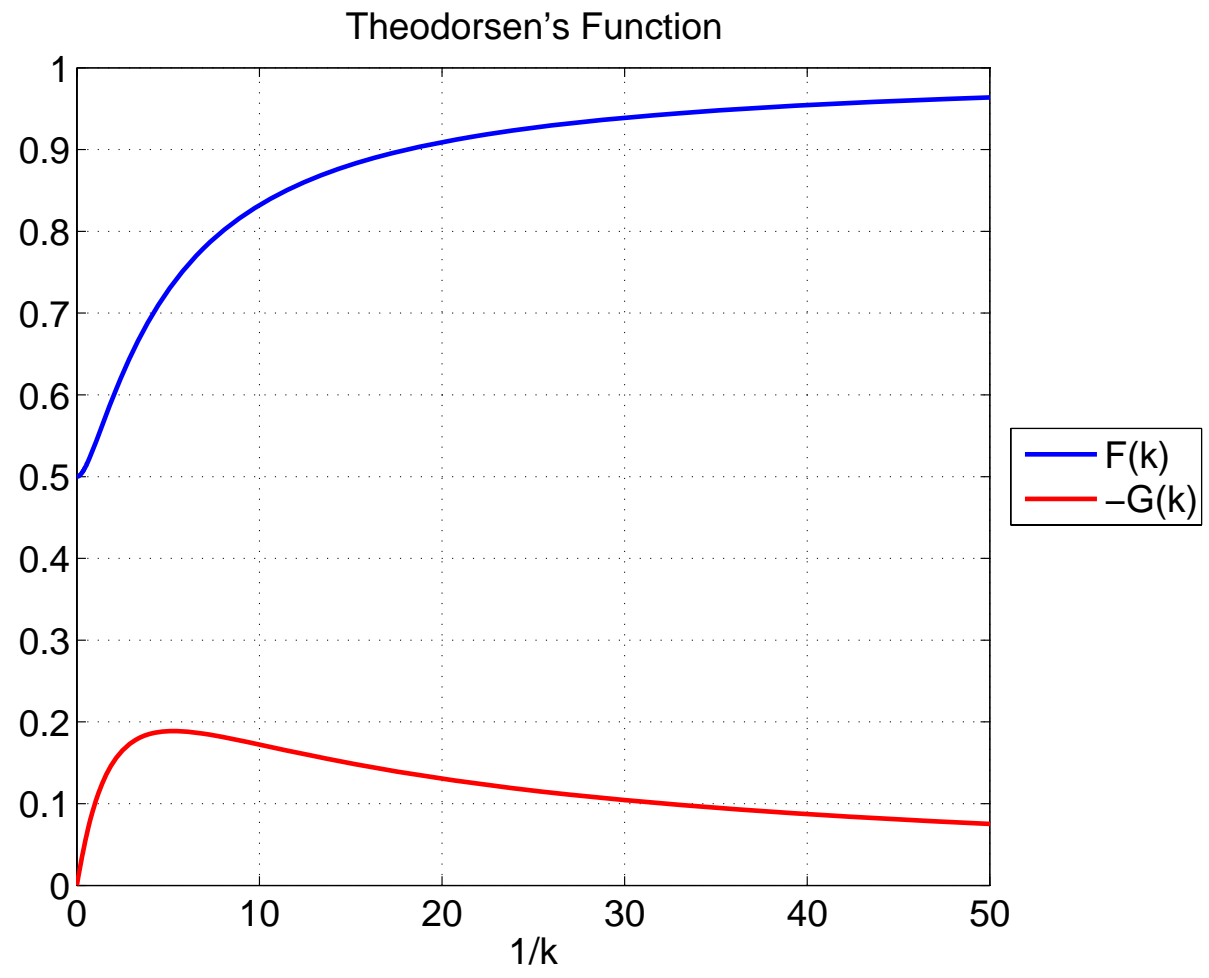

Figure A.1: Functions $\mathrm{F}$ and $\mathrm{G}$ as a function of $1 / k$ 